\title{
Probe split graphs
}

\author{
Van Bang Le and H.N. de Ridder
}

Universität Rostock, Institut für Informatik, Wissenschaftsbereich Theoretische Informatik, 18051 Rostock, Germany, \{le,hnridder\}@informatik.uni-rostock.de

received November 14, 2005, accepted July 19, 2007.

\begin{abstract}
An undirected graph $G=(V, E)$ is a probe split graph if its vertex set can be partitioned into two sets, $N$ (nonprobes) and $P$ (probes) where $N$ is independent and there exists $E^{\prime} \subseteq N \times N$ such that $G^{\prime}=\left(V, E \cup E^{\prime}\right)$ is a split graph. Recently Chang et al. gave an $O\left(V^{4}(V+E)\right)$ time recognition algorithm for probe split graphs. In this article we give $O\left(V^{2}+V E\right)$ time recognition algorithms and characterisations by forbidden induced subgraphs both for the case when the partition into probes and non-probes is given, and when it is not given.
\end{abstract}

Keywords: probe graphs, probe split, probe interval, graph class

In 1994, in the context of genome research, Zhang [15] introduced probe interval graphs. A graph is a probe interval graph if its vertex set can be partitioned into two sets, probes $P$ and non-probes $N$, such that $N$ is independent and new edges can be added between non-probes in such a way that the resulting graph is an interval graph. This definition can of course readily be generalized to some graph class $\mathcal{C}:$ A graph is probe $\mathcal{C}$ if its vertex set can be partitioned into two sets, probes $P$ and non-probes $N$, such that $N$ is independent and new edges can be added between non-probes in such a way that the resulting graph is in $\mathcal{C}$.

In this manner probe interval [15, 10, 12, 11], probe chordal [5, 3], including the intersection of probe chordal with weakly chordal graphs [5], the intersection of trees with probe interval graphs [14], the intersection of 2-trees with probe interval graphs [13], and probe interval bigraphs [2] have been defined and investigated. Moreover, already in 1989 - before Zhang's article — Hertz [7] defined what he called slim graphs, which are in fact probe Meyniel graphs, and proved that these are perfect. Hoang and Maffray [8] used Hertz' construction to define probe Gallai graphs. In the recent article [3] several probe classes are discussed, among them probe split graphs.

Given $\mathcal{C}$, the new graph class of all probe $\mathcal{C}$ graphs clearly contains $\mathcal{C}$ as a subclass. And when $\mathcal{C}$ is a subclass of $\mathcal{D}$, then so is probe $\mathcal{C}$ a subclass of probe $\mathcal{D}$. The general questions for new graph classes,

1. the existence of an efficient recognition algorithm, and

2. a characterisation of the structure, possibly by forbidden induced subgraphs,

exist in the case of probe $\mathcal{C}$ graphs in two variants: one where the partition into probes and non-probes is given and a more general one where it is not given, resulting in four problems.

For the class of probe interval graphs, the first recognition algorithm for the partitioned case was given in [10], and one with $O(V+E \log V)$ running time in [11], while recently the unpartitioned case has been solved [1]. Probe chordal graphs (unpartitioned) can be recognized in $O\left(V^{2} E\right)$ time [3]. A characterisation by forbidden induced subgraphs is open for both classes. A characterisation by forbidden induced 
subgraphs is known for the intersection of trees with probe interval graphs [14], both partitioned and unpartitioned, but for 2-trees it already seems to be a difficult problem [13]. The forbidden induced subgraph characterisation for the intersection of trees with probe interval graphs leads to a polynomial recognition algorithm, both partitioned and unpartitioned. Probe interval bigraphs can be recognized in polynomial time [2], a characterisation by forbidden subgraphs is unknown. Concerning probe split graphs, in [3] a polynomial recognition algorithm is sketched that can be implemented to run in time $O\left(V^{4}(V+E)\right)$, but the characterisation problem is not tackled.

From these results it is clear that neither recognizing nor characterising probe $\mathcal{C}$ graphs is a straightforward matter; in fact there is no positive characterisation result on any probe $\mathcal{C}$ class at all - the only positive result is on a subclass of probe interval graphs, the intersection of trees with probe interval graphs. In this article we will solve both the recognition and the characterisation problem for unpartitioned and partitioned probe split graphs.

All graphs considered are finite, undirected and simple. Given a graph $H$ and a positive integer $n$, $n H$ denotes the graph consisting of $n$ disjoint copies of $H . C_{n}, P_{n}$ and $K_{n}$ denote the chordless cycle, chordless path, and complete graph, respectively, on $n$ vertices. A chordless cycle on at least five vertices is called hole. Its complement is a anti-hole. An odd hole is a hole of odd length.

For two disjoint sets of vertices $X$ and $Y, X$ (1) $Y(X$ (0) $Y$ ) means that every vertex in $X$ is adjacent (nonadjacent) to every vertex in $Y$. Occasionally, when such use is clear from context, we may use (1), (0) as graph-valued operators: For graphs $X$ and $Y, X$ (0) $Y$ is the disjoint union of $X$ and $Y$ and $X$ (1) $Y$ results from $X$ (0) $Y$ by adding all possible edges with one endpoint in $X$ and the other in $Y$. We often identify a subset of vertices with the subgraph induced by that subset, and vice versa. A set of vertices is called independent or stable if the vertices are pairwise non-adjacent and it is called a clique if they are pairwise adjacent. Adjacency of two vertices $x, y$ in a graph $G=(V, E)$ is written $x y \in E$ or $x \sim y$. The (open) neighbourhood $N(v)$ of a vertex $v$ is the set of its neighbours, the closed neighbourhood $N[v]=N(v) \cup\{v\}$ and the non-neighbourhood $\bar{N}(v)=V \backslash N[v]$. Given a set of vertices $X \subseteq V$, the subgraph induced by $X$ is written $G[X]$. The complement of $G$ is written $\bar{G}$.

Recall that a graph is a split graph [4, 6] iff it can be partitioned into a clique and an independent set, and that the split graphs are precisely the $\left(2 K_{2}, C_{4}, C_{5}\right)$-free graphs.

Definition 1 A graph $G=(V, E)$ is a probe split graph if its vertex set $V$ can be partitioned into two sets, $N$ (non-probes) and $P$ (probes) where $N$ is independent and there exists $E^{\prime} \subseteq N \times N$ such that $G^{\prime}=\left(V, E \cup E^{\prime}\right)$ is a split graph.

As mentioned before, all split graphs are probe split. Bipartite graphs $G=(X \cup Y, E)$ are probe split as well: Take $N=X, P=Y$ and $E^{\prime}=N \times N$.

Theorem $2 G=(V, E)$ is a probe split graph iff its vertex set can be partitioned into three, possibly empty, sets $C, S, I$, such that $C$ is a clique, $S, I$ are independent sets and $C$ (1) $S$.

Proof: $(\Rightarrow)$ Let $G=(V, E)$ be a probe split graph with probes $P$ and non-probes $N(V=P \cup N)$. Let $G^{\prime}=\left(V, E \cup E^{\prime}\right)$, where $E^{\prime} \subseteq N \times N$, be a split graph with clique $K$ and independent set $U$. Now, let $C=K \cap P, S=K \cap N$ and $I=U$. In $G$ clearly $C$ is a clique, $S$ and $I$ are independent, and $C$ (1) $S$.

$(\Leftarrow)$ Let $G=(V, E)$ be a graph with a partition $(C, S, I)$ of $V$ into a clique $C$ and independent sets $S, I$ 
such that $C$ (1) $S$. Now, let $P=C \cup I, N=S$ and $E^{\prime}=N \times N$, then obviously $G^{\prime}=\left(V, E \cup E^{\prime}\right)$ is a split graph. Thus, $G$ is probe split.

From Th. 2 we see that probe split graphs are $(1,2)$-colourable and $P_{4}$-brittle (every $P_{4}$ has an endpoint in $I$ ) and therefore $P_{4}$-bipartite, perfectly orderable and perfect. (Definitions and references for all these classes can be found in ISGCI [9].)

Definition 3 A partition $(C, S, I)$ of the vertex set of a graph $G$ such that $C$ is a clique, $S$ and $I$ are independent sets and $C$ (1) $S$ is called a valid probe split partition of $G$.

By Th. 2 a graph is probe split iff it has a valid probe split partition. This fact will be used often and without further reference.

\section{Unpartitioned probe split graphs - An overview}

As every induced subgraph of a probe split graph is again a probe split graph, probe split graphs can characterized by forbidden induced subgraphs. In this section we will outline the proof that a graph is a probe split graph iff it has no induced subgraph from Fig. 1 on page 210. This will also give rise to a polynomial recognition algorithm for unpartitioned probe split graphs. The long and technical proof follows in the next section.

Theorem 4 A graph is a probe split graph iff it has no induced subgraph isomorphic to a graph in Fig.1.

Proof: $(\Rightarrow)$ By Lem.5

$(\Leftarrow)$ The proof is given in this and the next section.

Although the necessity of forbidding the graphs in Fig. 1 can easily be checked by brute force (i), the discussion in the next lemma will provide the reader with more insight in the structure of probe split graphs.

Lemma 5 The graphs in Fig. 1 are not probe split.

Proof: The key lies in the probe split partition from Th. 2. From this theorem we immediately get the following observations:

1. Every clique has at most one $I$-vertex, at most one $S$-vertex, the rest are $C$-vertices. In particular, every triangle contains at least one $C$-vertex.

2. Every $C_{4}$ consists either of alternating $S$ and $I$ vertices, or of two non-adjacent $S$-vertices, with the other two vertices being one $C$ and one $I$-vertex.

3. Combining these two, every house has an $I$-vertex as the roof, with one neighbour a $C$-vertex. From this the other vertices follow.

4. Every path on at least six vertices consists of alternating $S$ and $I$ vertices. If a path has even length, then precisely one of the end-vertices is an $S$-vertex.

(i) In fact, we verified by computer that the graphs in Fig. 1 are minimal non probe split graphs. 


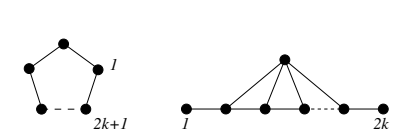

$\mathfrak{A}_{1}(k \geq 2)$

$\mathfrak{A}_{2}(k \geq 3)$

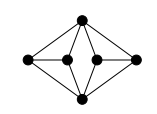

$\mathfrak{A}_{8}\left(\overline{C_{4} \cup P_{2}}\right)$

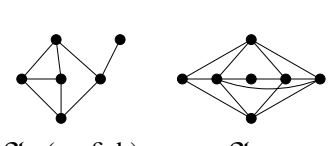

$\mathfrak{A}_{9}$ (co-fish)

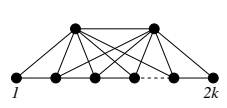

$\mathfrak{A}_{3}(k \geq 3)$

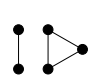

$\mathfrak{A}_{4}\left(K_{2} \cup K_{3}\right)$

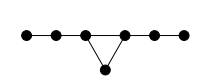

$\mathfrak{A}_{5}$ (longhorn)

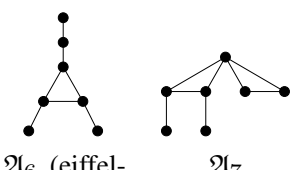

$\mathfrak{A}_{6}$ (eiffeltower)

$\mathfrak{A}_{7}$

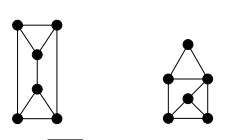

$\mathfrak{A}_{13}\left(\overline{C_{6}}\right)$

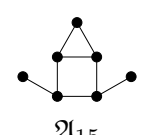

$\mathfrak{A}_{12}$ (co-
domino)

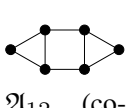

(antenna)

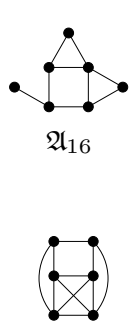

$\mathfrak{A}_{24}\left(\overline{2 P_{3}}\right)$
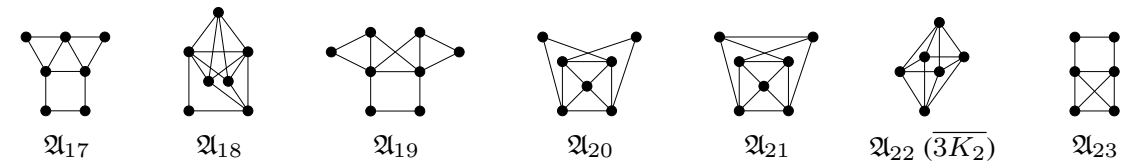

$\mathfrak{A}_{22}\left(\overline{3 K_{2}}\right)$

$\mathfrak{A}_{23}$

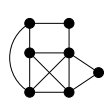

$\mathfrak{A}_{25}$
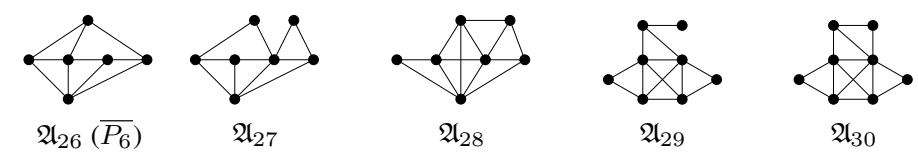

Fig. 1: Forbidden subgraphs for probe split graphs (continued on next page). 
5. Every $P_{5}$ either consists of alternating $S$ and $I$-vertices, or the middle vertex is a $C$-vertex, its neighbours $S$ - and the outer vertices $I$-vertices.

6. If the non-neighbourhood of a vertex contains an edge, then that vertex cannot be a $C$-vertex.

Obs. 1 gives $\mathfrak{A}_{4}\left(K_{2} \cup K_{3}\right)$ as forbidden subgraph, and together with Obs. $4 \mathfrak{A}_{5}$ (longhorn) If we take two triangles and unite one vertex of each triangle, we get a so-called butterfly. The vertex of degree four must be a $C$-vertex, while each wing consists of an $S$ and an $I$-vertex. This gives $\mathfrak{A}_{7}$ Obs. 2 gives $\mathfrak{A}_{8}$

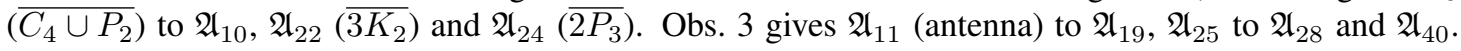
Obs. 4 gives $\mathfrak{A}_{2}, \mathfrak{A}_{3} \mid \mathfrak{A}_{35}$ and odd cycles of length at least 7 . Additionally a $C_{5}$ is forbidden because it cannot consist solely of $S$ and $I$ vertices, but if it contains a $C$ vertex, then its two non-neighbours in the $C_{5}$, which must be $I$-vertices, are adjacent to each other. This gives odd holes $\left.\sqrt{\mathfrak{A}_{1}}\right)$. Note that $\overline{\mathfrak{A}_{26}\left(\overline{P_{6}}\right)}$ forbids odd anti-holes of lenght at least 7, so probe split graphs are (odd hole, odd anti-hole)-free. Obs. 5 gives $\mathfrak{A}_{6}$ (eiffeltower)

Now consider the graph formed by $\mathfrak{A}_{29}$ with the vertex of degree one deleted. Since every vertex of degree two has two different non-neighbours in the $K_{4}$, and the $K_{4}$ has at most one $S$ and at most one $I$-vertex, it follows that the vertices of degree two must be $I$-vertices. This implies that the $K_{4}$ does not contain an $I$-vertex, and in particular that at least one of the lower two vertices of the $K_{4}$ must be a $C$-vertex. Hence every neighbour of the highest vertex of degree two must be adjacent to this $C$-vertex. This gives $\mathfrak{A}_{29}, \mathfrak{A}_{30}, \mathfrak{A}_{31}$.

From Obs. 2 we have that a vertex $v$ that is universal to a $C_{4}$ is a $C$-vertex. Vertices that are nonadjacent to $v$ then must be $I$-vertices, and their neighbours in the $C_{4}$ are in $S$ or $C$. This leads to $\mathfrak{A}_{20}$, $\mathfrak{A}_{21}, \mathfrak{A}_{32}$ to $\mathfrak{A}_{34}, \mathfrak{A}_{36}$ to $\mathfrak{A}_{39}$ and $\mathfrak{A}_{41}$ to $\mathfrak{A}_{45}$.

Finally to graphs $\sqrt[\mathfrak{A}_{46}]{ }$ to $\mathfrak{A}_{52}$. A vertex that is not part of the $P_{3}$ (1) $P_{3}$ must be an $I$-vertex (Obs. 6- it is easy to see that they cannot be $S$-vertices), and thus the $P_{3}$ s do not contain any $I$-vertices. But every $P_{3}$ contains at least one non- $C$-vertex, i.c. an $S$-vertex. These $S$-vertices are adjacent.

The sufficiency of Th. 4 is proved inductively. Let $G$ have no induced subgraphs isomorphic to a graph listed in Fig. 1] Set

$$
\begin{aligned}
& B=\{v \in V(G) \mid \bar{N}(v) \text { is not a stable set }\} \\
& C=\{v \in V(G) \mid N(v) \text { does not induce a split graph }\}
\end{aligned}
$$

Proposition 6 Assuming $G=(V, E)$ does not contain any graph listed in Fig. 1 as an induced subgraph, then
a) $B \cap C=\emptyset$;
b) $C$ is a clique;
c) $G[B]$ is bipartite.

Proposition 7 Assuming $G=(V, E)$ does not contain any graph listed in Fig. 1 as an induced subgraph, then for every nontrivial (i.e. with at least two vertices) connected component $B^{\prime}=\left(X \cup Y, E^{\prime}\right)$ of $G[B]$, $X$ (1) $C$ or $Y$ (1) $C$.

The basis of the induction is the case that $V=B \cup C$. In this case, the Prop. 7 states how to define a valid probe split partition $(C, S, B \backslash S)$ of $G$ : For every non-trivial component $B^{\prime}=\left(X \cup Y, E^{\prime}\right)$ of $B$, let $S\left(B^{\prime}\right) \in\{X, Y\}$ with $S\left(B^{\prime}\right)$ (1) $C$ and define $S=\bigcup_{B^{\prime}} S\left(B^{\prime}\right)$. 


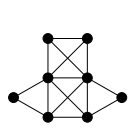

$\mathfrak{A}_{31}$

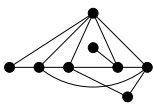

$\mathfrak{A}_{37}$

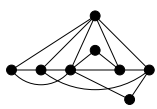

$\mathfrak{A}_{43}$
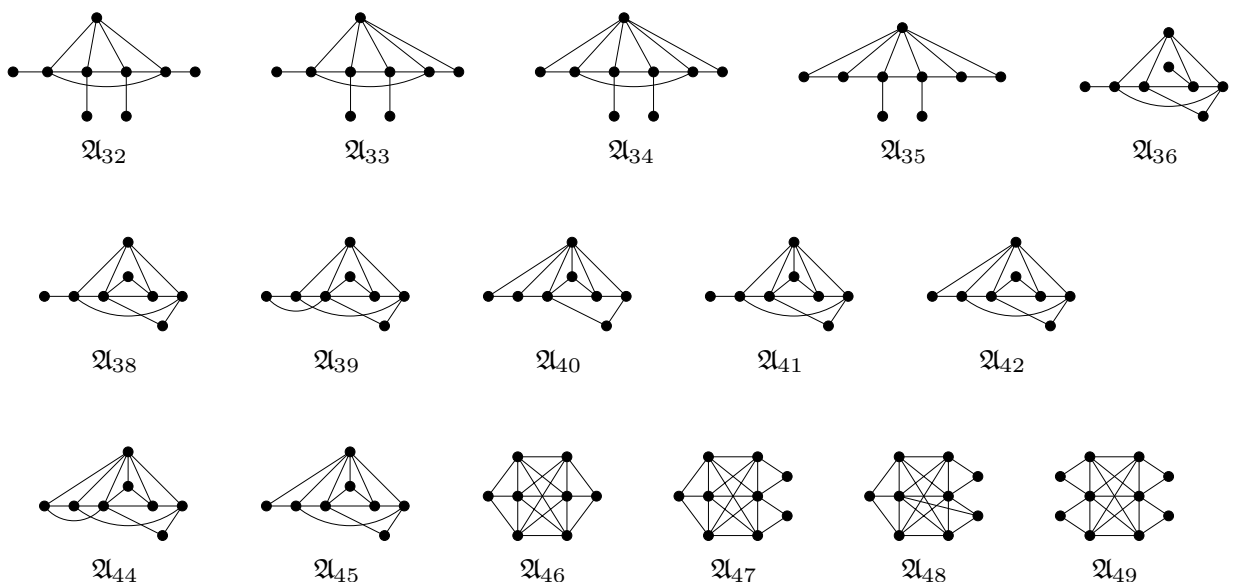

$\mathfrak{A}_{45}$
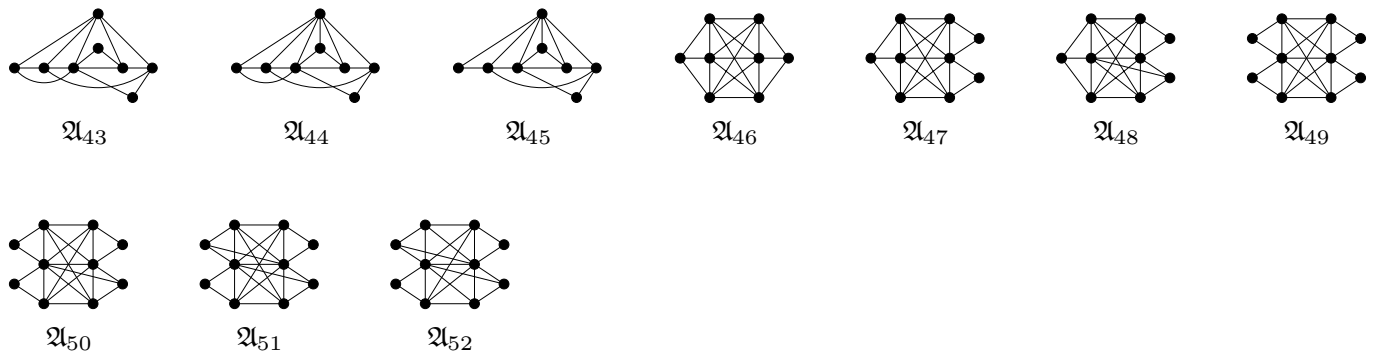

Fig. 1: Forbidden subgraphs for probe split graphs (continuation of previous page).

The induction step occurs when $V \neq B \cup C$. According to Prop. 8, we can remove a vertex $v \in$ $V \backslash(B \cup C)$ and inductively build a probe split partition on a valid partition of $G-v$ :

Proposition 8 Assuming $G=(V, E)$ does not contain any graph listed in Fig. 1 1 as an induced subgraph, then for every vertex $v \in V \backslash(B \cup C)$ : If $G-v$ is a probe split graph, then so is $G$. Moreover, a valid partition of $G$ can be obtained from a valid partition of $G-v$ in linear time.

Using Prop. 6, 7 and 8 we can give Alg. 1 to recognize probe split graphs.

Theorem 9 Algorithm 1 correctly recognizes probe split graphs, and can be implemented with running time $O\left(V^{2}+V E\right)$.

Proof: Correctness follows from Lem.5. Prop.6 6 Prop. 7 and Prop. 8 It is obvious that calculating $B$ and $C$ and verifying Prop.6 and Prop.7(first if-statement) can be done in the desired timebound. The next two if-statement form a recursive algorithm. Note that in the recursion we do not need to calculate $B, C$, nor to verify Prop. 6 and Prop. 7 . Let $n=|V \backslash(B \cup C)|$, then the basis of the recursion (second if-statement) can be executed in time $T(0)=O(V+E)$, and the third if-statement takes $T(n)=T(n-1)+O(V+E)$ by Prop. 8 . Solving the recurrence gives $O\left(V^{2}+V E\right)$. 


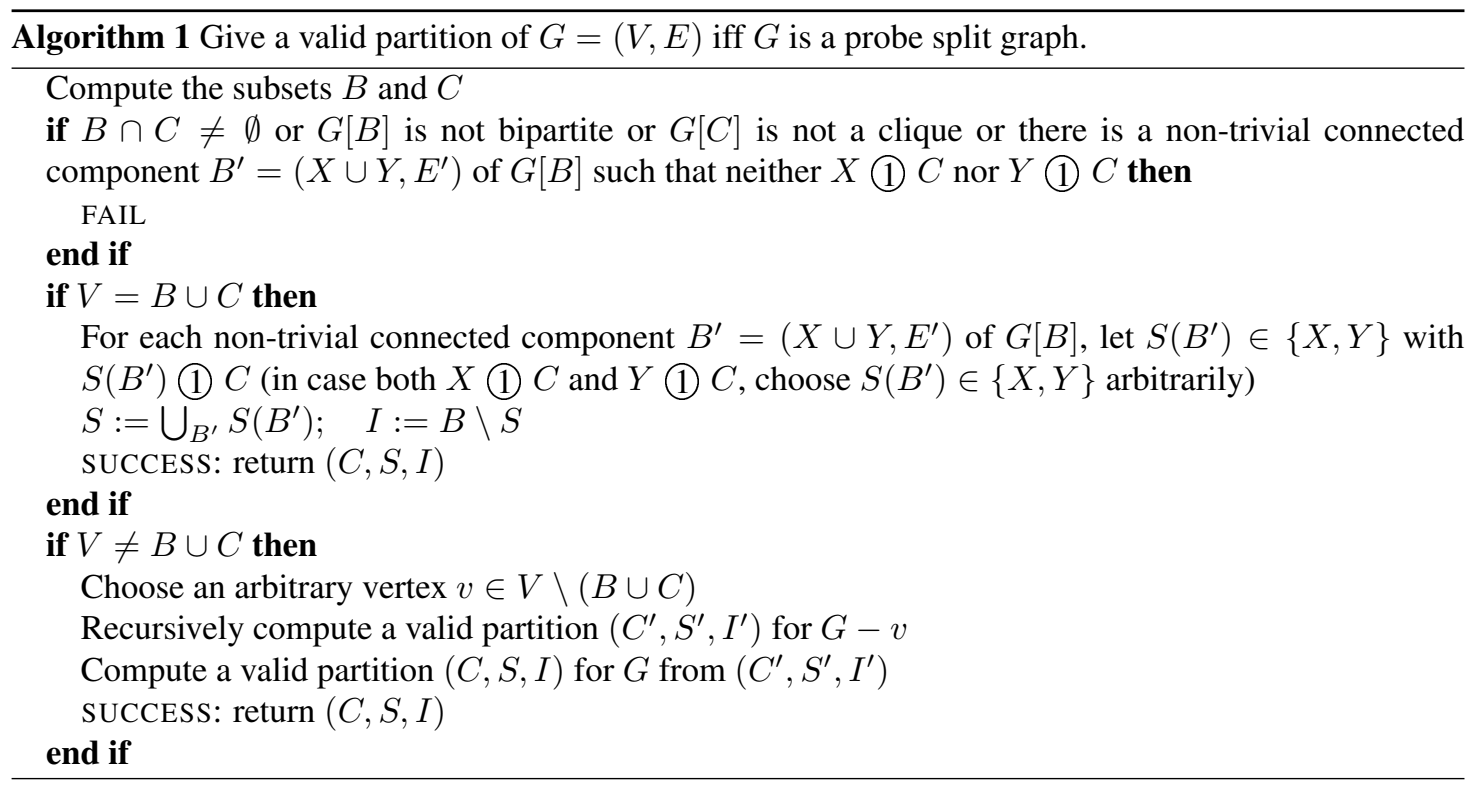

\section{Unpartitioned probe split graphs - The proof of Prop. 6, 7 and 8}

In this section we prove Prop. 6 . Prop.7 7 and Prop. 8 . We assume that $G=(V, E)$ has no induced subgraph from Fig. 1 and that $B, C \subseteq V$ are as defined in the previous section.

\subsection{The proof of Proposition 6}

Lemma 10 (Prop. 6.a) $B \cap C=\emptyset$.

Proof: Assume to the contrary that there exists a vertex $v \in B \cap C$. As $v \in B$, there exist adjacent vertices $x, y \in \bar{N}(v)$. As $v \in C$ and $G$ is $C_{5}$-free, there exist vertices $v_{1}, v_{2}, v_{4}, v_{4} \in N(v)$ such that $\left\{v_{1}, v_{2}, v_{3}, v_{4}\right\}$ induces a $2 K_{2}$ with edges $v_{1} v_{2}$ and $v_{3} v_{4}$, or the $C_{4}=\left(v_{1}, v_{2}, v_{3}, v_{4}, v_{1}\right)$.

We consider the first case. In this case, each of $x, y$ is adjacent to at most two of the vertices $v_{1}, v_{2}, v_{3}, v_{4}$ otherwise $x$ or $y$ together with $v_{1}, v_{2}, v_{3}, v_{4}$ and $v$ would induce a $\mathfrak{A}_{14}$ or $\mathfrak{A}_{8}\left(\overline{C_{4} \cup P_{2}}\right)$ Moreover, $x$ (respectively, $y$ ) cannot be adjacent to both $v_{1}, v_{2}$, or to both $v_{3}, v_{4}$. For, if $x$ is adjacent to both $v_{1}, v_{2}$ then $x, y, v_{3}, v_{4}$ and $v$ would induce a $\mathfrak{A}_{4}\left(K_{2} \cup K_{3}\right)$ (if $\left.y \not v_{3}, v_{4}\right)$, or $y, x, v_{1}, v$ and $v_{3}$ or $v_{4}$ would induce a $C_{5}$ (if $y \sim v_{3}$ or $y \sim v_{4}$ ). So, let without loss of generality, $x$ be nonadjacent to $v_{1}$ and $v_{3}$.

Now, if $y$ is nonadjacent to all $v_{i}, i=1,2,3,4$, then $x, y, v, v_{1}, v_{2}, v_{3}, v_{4}$ induce a $\mathfrak{A}_{16}$ (if $x \sim v_{2}, v_{4}$ ), or $\left\{x, y, v, v_{1}, v_{2}\right\}$ or $\left\{x, y, v, v_{3}, v_{4}\right\}$ induce a $\mathfrak{A}_{4}\left(K_{2} \cup K_{3}\right)$ (otherwise).

If $y$ is adjacent to exactly one of the $v_{i}$, then we may assume without loss of generality that $y$ is nonadjacent to $v_{1}, v_{2}$. Then $x$ must be adjacent to $v_{2}$ otherwise $x, y, v, v_{1}, v_{2}$ would induce a $\mathfrak{A}_{4}\left(K_{2} \cup K_{3}\right)$. Hence $y$ and $v_{3}$ are nonadjacent otherwise $x, y, v, v_{2}, v_{3}$ would induce a $C_{5}$. It follows that $y$ is adjacent to $v_{4}$, and $x, y, v, v_{2}, v_{4}$ induce a $C_{5}$ (if $x \not v_{4}$ ), or $x, y, v, v_{1}, v_{2}, v_{4}$ induce a $\mathfrak{A}_{12}$ (co-domino)

Finally, let $y$ be adjacent to (exactly) two of the $v_{i}$. If $y$ is adjacent to $v_{1}$ and $v_{3}$ then $x, y, v_{1}, v_{2}, v_{3}, v_{4}$ and $v$ induce a $\mathfrak{A}_{16}\left(\right.$ if $x \not v_{2}, v_{4}$ ), or $x, y, v, v_{1}, v_{4}$ or $x, y, v, v_{2}, v_{3}$ induce a $C_{5}$ (if $x \sim v_{4}$ or $x \sim v_{2}$ ). If 
$y$ is adjacent to $v_{1}$ and $v_{4}$ then $x, y, v, v_{1}, v_{2}, v_{3}, v_{4}$ induce a $\mathfrak{A}_{16}\left(\right.$ if $\left.x \nsim v_{2}, v_{4}\right)$, or $x, y, v, v_{2}, v_{4}$ induce a $C_{5}$ (if $x \sim v_{2}, x \nsim v_{4}$ ), or $x, y, v, v_{1}, v_{2}, v_{3}, v_{4}$ induce a $\mathfrak{A}_{12}$ (co-domino) (if $x \nsim v_{2}, x \sim v_{4}$ ) or $x, y, v, v_{1}, v_{2}, v_{4}$ induce a $\mathfrak{A}_{13}\left(\overline{C_{6}}\right)$. The first case is settled.

We consider the case of the $C_{4}=\left(v_{1}, v_{2}, v_{3}, v_{4}, v_{1}\right)$. In this case, each of $x, y$ is adjacent to at least two of the vertices $v_{1}, v_{2}, v_{3}, v_{4}$ otherwise there would be a $\mathfrak{A}_{4}\left(K_{2} \cup K_{3}\right)$ or a $C_{5}$, but to at most three of the $v_{i}$ otherwise there would be a $\overline{\mathfrak{A}_{22}\left(\overline{3 K_{2}}\right)}$

Let $x$ be adjacent to $v_{1}, v_{2}, v_{3}$. If $y$ is also adjacent to $v_{1}, v_{2}, v_{3}$ then $x, y, v, v_{1}, v_{3}, v_{4}$ induce a $\mathfrak{A}_{8}$ $\left.\overline{\left(C_{4} \cup P_{2}\right.}\right)$ If $y$ is adjacent to $v_{1}, v_{2}, v_{4}$ or to $v_{2}, v_{3}, v_{4}$ then $x, y, v, v_{1}, v_{3}, v_{4}$ induce a $\mathfrak{A}_{26}\left(\overline{P_{6}}\right)$. If $y$ is adjacent to $v_{1}, v_{3}, v_{4}$ then $x, y, v, v_{2}, v_{4}$ induce a $C_{5}$. If $y$ is adjacent to (exactly) $v_{1}, v_{2}$, or to $v_{2}, v_{3}$ then $x, y, v, v_{1}, v_{3}, v_{4}$ induce a $\mathfrak{A}_{14}$ If $y$ is adjacent to (exactly) $v_{2}, v_{4}$ then $x, y, v, v_{1}, v_{2}, v_{4}$ induce a $\overline{\mathfrak{A}_{26}\left(\overline{P_{6}}\right)}$. If $y$ is adjacent to $v_{1}, v_{4}$ (or to $v_{3}, v_{4}$ ) then $x, y, v, v_{2}, v_{4}$ induce a $C_{5}$. Thus, $x$, and by symmetry, $y$ must be adjacent to exactly two of the $v_{i}$.

We first consider the case that at least one of $x, y$ is adjacent to (exactly) two non-neighbours in $\left\{v_{1}, \ldots, v_{4}\right\}$. Without loss of generality, assume $x$ is adjacent to $v_{1}, v_{3}$. If $y$ is adjacent to $v_{2}, v_{4}$ then

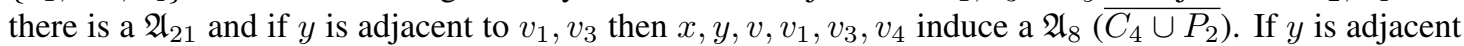
to $v_{1}, v_{2}$ or to $v_{2}, v_{3}$, then $x, y, v, v_{1}, v_{3}, v_{4}$ induce a $\mathfrak{A}_{14}$ and if $y$ is adjacent to $v_{3}, v_{4}$ or to $v_{4}, v_{1}$, then $x, y, v, v_{1}, v_{2}, v_{3}$ induce a $\mathfrak{A}_{14}$.

Finally, consider the case that each of $x, y$ is adjacent to two neighbours in $\left\{v_{1}, \ldots, v_{4}\right\}$. Without loss of generality, let $x$ be adjacent to $v_{1}, v_{2}$. If $y$ is adjacent to $v_{1}, v_{4}$ or to $v_{2}, v_{3}$, then $x, y, v_{2}, v_{3}, v_{4}$ or $x, y, v_{1}, v_{3}, v_{4}$, respectively, induce a $C_{5}$, and if $y$ is adjacent to $v_{3}, v_{4}$, then $x, y, v_{1}, v_{2}, v_{3}, v_{4}$ induce a $\mathfrak{A}_{13}\left(\overline{C_{6}}\right)$

Lemma 11 (Prop. 6.b) $C$ is a clique.

Proof: Assume to the contrary that there exist two nonadjacent vertices $c_{1} \neq c_{2}$ in $C$. By Prop. $6 . \mathrm{a}, \bar{N}\left(c_{1}\right)$ and $\bar{N}\left(c_{2}\right)$ are independent. This implies that every vertex that is (not) adjacent to $c_{1}$ is also (not) adjacent to $c_{2}$ and vice versa. Thus, $N\left(c_{1}\right)=N\left(c_{2}\right)$. Now, as $c_{1} \in C$ and because $G$ is $C_{5}$-free, $G\left[N\left(c_{1}\right)\right]$

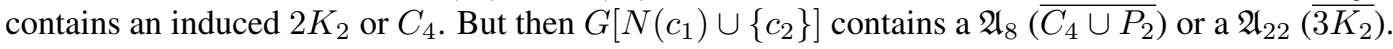

Lemma 12 (Prop. 6.c) $G[B]$ is bipartite.

Proof: Assume to the contrary that $B$ does not induce a bipartite graph. As $G$ is odd hole-free, $B$ contains a triangle $v_{1} v_{2} v_{3}$. As $v_{i} \in B$, there exist adjacent vertices $x_{i}, y_{i}$ in $\bar{N}\left(v_{i}\right), i=1,2,3$. In the following claims, $i, j, k$ are arbitrarily chosen with $\{i, j, k\}=\{1,2,3\}$.

Claim $1 N\left(v_{i}\right) \cap\left\{x_{j}, y_{j}, x_{k}, y_{k}\right\} \neq \emptyset$.

PROOF: Assume to the contrary that $N\left(v_{i}\right) \cap\left\{x_{j}, y_{j}, x_{k}, y_{k}\right\}=\emptyset$. As $G$ has no induced $\mathfrak{A}_{4}\left(K_{2} \cup K_{3}\right)$, $v_{j} \sim x_{k}$ or $v_{j} \sim y_{k}$, but not both otherwise $v_{i}, v_{k}, x_{k}, y_{k}$ would induce a $2 K_{2}$ in $N\left(v_{j}\right)$ and $v_{j}$ would belong to $C$, contradicting Prop.6.a. Likewise, $v_{k}$ is adjacent to exactly one of $x_{j}, y_{j}$.

Let, without loss of generality, $v_{j} \sim x_{k}$ and $v_{k} \sim x_{j}$. Then $x_{j} \notin\left\{x_{k}, y_{k}\right\}$ and $x_{k} \notin\left\{x_{j}, y_{j}\right\}$. Moreover, $y_{j} \neq y_{k}$ otherwise $v_{j}, v_{k}, x_{j}, y_{j}, x_{k}$ would induce a $C_{5}$ (if $x_{j} \nsim x_{k}$ ) or $v_{i}, v_{j}, v_{k}, x_{j}, y_{j}, x_{k}$ would induce a $\mathfrak{A}_{12}$ (co-domino) (if $x_{j} \sim x_{k}$ ). Hence $y_{j} \not y_{k}$ otherwise $y_{j}, y_{k}, v_{i}, v_{j}, v_{k}$ would induce a $\mathfrak{A}_{4}\left(K_{2} \cup K_{3}\right)$, and $y_{j} \not x_{k}, y_{k} \nsim x_{j}$ otherwise there would be a $C_{5}$ or a $\overline{\mathfrak{A}_{12} \text { (co-domino) }}$ Now, $v_{i}, v_{j}, v_{k}, x_{j}, y_{j}, x_{k}, y_{k}$ induce a $\mathfrak{A}_{15}$ (if $x_{j} \sim x_{k}$ ) or a $\mathfrak{A}_{5}$ (longhorn) (otherwise). 
Claim $2 x_{i}, y_{i}, v_{j}, v_{k}$ do not induce a $C_{4}$.

PROOF: Assume to the contrary that $x_{i}, y_{i}, v_{j}, v_{k}$ do induce a $C_{4}$, say $v_{j} \sim x_{i}, v_{k} \sim y_{i}$ and $v_{j} \not y_{i}$, $v_{k} \not x_{i}$. We first prove some observations.

$$
N\left(v_{i}\right) \backslash N\left(v_{j}\right) \subseteq N\left(v_{k}\right) \backslash N\left(y_{i}\right)
$$

Proof of (1): Let $v \in N\left(v_{i}\right) \backslash N\left(v_{j}\right)$. First assume $v \not v_{k}$. Then $v \sim y_{i}$ or $v \sim x_{i}$ (otherwise there would be a $\mathfrak{A}_{11}$ (antenna), but not both (otherwise there would be a $\mathfrak{A}_{13}\left(\overline{C_{6}}\right)$. . Now, $v_{i}, x_{i}, y_{i}, v$ and $v_{j}$ (if $v \sim y_{i}$ ) or $v_{k}$ (if $v \sim x_{i}$ ) induce a $C_{5}$. Thus, $v \sim v_{k}$. Next, assume $v \sim y_{i}$. Then $v \sim x_{i}$, too (otherwise there would be a $C_{5}$ ). Hence $v_{i}, v_{j}, v_{k}, x_{i}, y_{i}, v$ induce a $\mathfrak{A}_{26}\left(\overline{P_{6}}\right)$ Thus, $v \not y_{i}$, and (1) follows.

By symmetry, we have:

$$
N\left(v_{i}\right) \backslash N\left(v_{k}\right) \subseteq N\left(v_{j}\right) \backslash N\left(x_{i}\right)
$$

We next show:

$$
N\left(v_{i}\right) \backslash N\left(v_{j}\right) \neq \emptyset \Rightarrow \bar{N}\left(v_{k}\right) \subseteq \bar{N}\left(v_{i}\right)
$$

Proof of (3): Let $v \in N\left(v_{i}\right) \backslash N\left(v_{j}\right)$. By (1), $v \sim v_{k}, v \not y_{i}$. Consider a vertex $u \in \bar{N}\left(v_{k}\right)$, and assume $u \sim v_{i}$. Then, by (2), $u \sim v_{j}, u \neq x_{i}$. Moreover, $v \nsim u$ otherwise $v_{i}$ would belong to $C$, contradicting Prop.6.a.

Now, if $u \neq y_{i}$ and $v \not x_{i}$ then $v_{i}, v_{j}, v_{k}, x_{i}, y_{i}, u, v$ induce a $\mathfrak{A}_{17}$. If $u \sim y_{i}$ and $v \sim x_{i}$ then $v_{i}, x_{i}, y_{i}, u, v$ induce a $C_{5}$. If $u \sim y_{i}$ and $v \not x_{i}$ (or $u \neq y_{i}$ and $v \sim x_{i}$ ) then $v_{i}, x_{i}, y_{i}, u, v$ and $v_{j}$ (respectively, $v_{k}$ ) induce a $\mathfrak{A}_{11}$ (antenna). Thus, $u \not v_{i}$, and $(3)$ follows.

By symmetry, we have:

$$
N\left(v_{i}\right) \backslash N\left(v_{k}\right) \neq \emptyset \Rightarrow \bar{N}\left(v_{j}\right) \subseteq \bar{N}\left(v_{i}\right)
$$

Now, by Claim 1 and symmetry, $v_{i}$ is adjacent to a vertex $v \in\left\{x_{j}, y_{j}\right\}$. By (1), $v \sim v_{k}, v \not$ $y_{i}$. In particular, $v \notin\left\{x_{k}, y_{k}\right\}$. By (3), $x_{k}$ and $y_{k}$ are nonadjacent to $v_{i}$, hence $v_{j} \sim x_{k}$ or $v_{j} \sim y_{k}$ otherwise there would be a $\mathfrak{A}_{4}\left(K_{2} \cup K_{3}\right)$, but not both otherwise $v_{j}$ would belong to $C$, contradicting Prop. 6.a.

Let, without loss of generality, $v_{j} \sim x_{k}$. Then $v \sim x_{k}$ otherwise $v_{i}, v_{k}, v, x_{k}, y_{k}$ would induce a $\mathfrak{A}_{4}$ $\left(K_{2} \cup K_{3}\right)$ (if $v \not y_{k}$ ) or $v_{i}, v, v_{j}, x_{k}, y_{k}$ would induce a $C_{5}$ (if $v \sim y_{k}$ ). Now, $v_{i}, v_{j}, v_{k}, v, x_{k}, y_{k}$ induce a $\mathfrak{A}_{9}$ (co-fish) (if $v \not y_{k}$ ) or a $\mathfrak{A}_{14}$ (if $v \sim y_{k}$ ). This final contradiction proves Claim 2

CLAIM 3 If $x_{j}, y_{j} \in N\left(v_{i}\right)$ then $N\left(v_{k}\right) \cap\left\{x_{j}, y_{j}\right\} \neq \emptyset,\left|N\left(v_{k}\right) \cap\left\{x_{i}, y_{i}\right\}\right|=1$, and $N\left(v_{j}\right) \cap\left\{x_{i}, y_{i}\right\}=\emptyset$. PROOF: Note first that $x_{i}, y_{i}, x_{j}, y_{j}$ are pairwise distinct, since $x_{j}, y_{j} \in N\left(v_{i}\right)$. From $v_{i} \in B$ and Prop. 6.a it follows that $N\left(v_{k}\right) \cap\left\{x_{j}, y_{j}\right\} \neq \emptyset$. Let $v_{k} \sim x_{j}$, say.

Suppose that $\left|N\left(v_{k}\right) \cap\left\{x_{i}, y_{i}\right\}\right| \neq 1$. If $x_{i} \sim v_{k}$ and $y_{i} \sim v_{k}$ then $G\left[N\left(v_{k}\right)\right]$ contains an induced $C_{5}, C_{4}$, or $2 K_{2}$, hence $v_{k} \in C$, contradicting Prop.6.a Thus, $N\left(v_{k}\right) \cap\left\{x_{i}, y_{i}\right\}=\emptyset$. Then $v_{j} \sim x_{i}$ or $v_{j} \sim y_{i}$ (otherwise $v_{i}, v_{j}, v_{k}, x_{i}, y_{i}$ would induce a $\mathfrak{A}_{4}\left(K_{2} \cup K_{3}\right)$ ), but not both (otherwise $v_{j}$ would belong to $C$, contradicting Prop. 6.a). Let $v_{j} \sim x_{i}$, say. Also, $x_{j} \sim x_{i}$ or $x_{j} \sim y_{i}$ (otherwise $x_{j}, v_{i}, v_{k}, x_{i}, y_{i}$ would induce a $\mathfrak{A}_{4}\left(K_{2} \cup K_{3}\right)$, but not both (otherwise $v_{i}, v_{j}, v_{k}, x_{i}, y_{i}, x_{j}$ would 
induce a $\mathfrak{A}_{14}$. Now, if $x_{j} \sim x_{i}$ then $v_{i}, v_{j}, v_{k}, x_{i}, y_{i}, x_{j}$ induce a $\mathfrak{A}_{9}$ (co-fish) and if $x_{j} \sim y_{i}$ then $v_{i}, v_{j}, x_{i}, y_{i}, x_{j}$ induce a $C_{5}$. It follows that $\left|N\left(v_{k}\right) \cap\left\{x_{i}, y_{i}\right\}\right|=1$.

Let, without loss of generality, $x_{i} \sim v_{k}$. Next we suppose that $N\left(v_{j}\right) \cap\left\{x_{i}, y_{i}\right\} \neq \emptyset$. If $x_{i} \sim v_{j}$ then $x_{i} \nsim x_{j}$ (otherwise $v_{k}$ would belong to $C$, contradicting Prop. 6.a), and $y_{i} \nsim x_{j}$ (otherwise $v_{i}, v_{j}, v_{k}, x_{i}, y_{i}, x_{j}$ would induce a $\overline{\mathfrak{A}_{26}\left(\overline{P_{6}}\right)}$ (if $y_{i} \sim v_{j}$ ) or $x_{i}, y_{i}, x_{j}, v_{i}, v_{j}$ induce a $C_{5}$ (if $y_{i} \not$ $\left.\left.v_{j}\right)\right)$. Now, if $y_{j} \nsim x_{i}$ and $y_{j} \nsim y_{i}$ then $v_{i}, x_{i}, y_{i}, x_{j}, y_{j}$ induce a $\mathfrak{A}_{4}\left(K_{2} \cup K_{3}\right)$ If $y_{j} \sim x_{i}$ and $y_{j} \sim y_{i}$ then $v_{i}, v_{k}, x_{i}, y_{i}, x_{j}, y_{j}$ induce a $\mathfrak{A}_{14}$ If $y_{j} \sim x_{i}$ and $y_{j} \nsim y_{i}$ then $v_{i}, v_{k}, x_{i}, y_{i}, x_{j}, y_{j}$ induce a $\mathfrak{A}_{9}$ (co-fish), and finally, if $y_{j} \nsim x_{i}$ and $y_{j} \sim y_{i}$ then $x_{i}, y_{i}, x_{j}, y_{j}, v_{k}$ induce a $C_{5}$. Thus, $x_{i} \nsim v_{j}$. By Claim 2, therefore, $y_{i} \nsim v_{j}$, and it follows that $N\left(v_{j}\right) \cap\left\{x_{i}, y_{i}\right\}=\emptyset$.

CLAIM $4\left|N\left(v_{i}\right) \cap\left\{x_{j}, y_{j}\right\}\right| \leq 1$

Proof: Assume to the contrary that $x_{j} \sim v_{i}$ and $y_{j} \sim v_{i}$. By Claim $3 . N\left(v_{j}\right) \cap\left\{x_{i}, y_{i}\right\}=\emptyset$ and we may assume that $v_{k} \sim x_{j}, v_{k} \sim x_{i}$, and $v_{k} \not y_{i}$.

By Claim 1, $N\left(v_{j}\right) \cap\left\{x_{k}, y_{k}\right\} \neq \emptyset$. If $x_{k}, y_{k} \in N\left(v_{j}\right)$ then, by Claim $3, N\left(v_{k}\right) \cap\left\{x_{j}, y_{j}\right\}=\emptyset$, a contradiction. Thus, $\left|N\left(v_{j}\right) \cap\left\{x_{k}, y_{k}\right\}\right|=1$, say $v_{j} \sim x_{k}$ and $v_{j} \not y_{k}$. Moreover, $\mid N\left(v_{i}\right) \cap$ $\left\{x_{k}, y_{k}\right\} \mid \leq 1$, otherwise, by Claim 3 again, $N\left(v_{k}\right) \cap\left\{x_{i}, y_{i}\right\}=\emptyset$, a contradiction.

Suppose $\left|N\left(v_{i}\right) \cap\left\{x_{k}, y_{k}\right\}\right|=1$. Then by Claim 2, $v_{i} \sim x_{k}$. In particular, $x_{k} \notin\left\{x_{i}, y_{i}\right\}$, and $x_{k} \sim x_{i}$ or $x_{k} \sim y_{i}$ otherwise $v_{i}, v_{j}, x_{k}, x_{i}, y_{i}$ would induce a $\mathfrak{A}_{4}\left(K_{2} \cup K_{3}\right)$, but not both otherwise $v_{i}, v_{j}, v_{k}, x_{k}, x_{i}, y_{i}$ would induce a $\mathfrak{A}_{14}$ Now, $x_{k}, y_{i}, x_{i}, v_{k}, v_{i}$ induce a $C_{5}$ (if $x_{k} \not x_{i}$ ) or $v_{i}, v_{j}, v_{k}, x_{k}, x_{i}, y_{i}$ induce a $\mathfrak{A}_{9}$ (co-fish) (if $x_{k} \sim x_{i}$ ).

Thus, $N\left(v_{i}\right) \cap\left\{x_{k}, y_{k}\right\}=\emptyset$. Then $x_{j} \notin\left\{x_{k}, y_{k}\right\}$, and the same arguments above applied for $v_{i}, v_{j}, v_{k}, x_{j}, x_{k}, y_{k}$ show again that there exists a $\mathfrak{A}_{4}\left(K_{2} \cup K_{3}\right)$ or a $\mathfrak{A}_{14}$ or a $C_{5}$, or a $\mathfrak{A}_{9}$ (cofish) Claim 4 is proved.

Now, as $G$ has no induced $\mathfrak{A}_{4}\left(K_{2} \cup K_{3}\right)$, and because of Claims 2 and 4 , we have the following two cases with a suitable choice of $i, j, k$.

Case $1 N\left(v_{j}\right) \cap\left\{x_{i}, y_{i}\right\}=N\left(v_{k}\right) \cap\left\{x_{i}, y_{i}\right\}$ and $N\left(v_{k}\right) \cap\left\{x_{j}, y_{j}\right\}=N\left(v_{i}\right) \cap\left\{x_{j}, y_{j}\right\}$.

Then we may assume that $N\left(v_{j}\right) \cap\left\{x_{i}, y_{i}\right\}=N\left(v_{k}\right) \cap\left\{x_{i}, y_{i}\right\}=\left\{x_{i}\right\}$ and $N\left(v_{k}\right) \cap\left\{x_{j}, y_{j}\right\}=$ $N\left(v_{i}\right) \cap\left\{x_{j}, y_{j}\right\}=\left\{x_{j}\right\}$. It follows that $x_{i} \notin\left\{x_{j}, y_{j}\right\}, x_{j} \notin\left\{x_{i}, y_{i}\right\}$. As $v_{k} \in B$ and because of Prop.6.a $x_{i} \nsim x_{j}$. Moreover, $x_{i} \nsim y_{j}$ (otherwise $x_{i}, y_{j}, x_{j}, v_{i}, v_{j}$ would induce a $C_{5}$ ), $x_{j} \nsim y_{i}$ (otherwise $x_{j}, y_{i}, x_{i}, v_{j}, v_{i}$ would induce a $C_{5}$ ). In particular, $y_{i} \neq y_{j}$.

Now, if $y_{i} \sim y_{j}$ then $y_{i}, y_{j}, x_{j}, v_{k}, x_{i}$ induce a $C_{5}$. If $y_{i} \nsim y_{j}$ then $v_{i}, v_{j}, v_{k}, x_{i}, y_{i}, x_{j}, y_{j}$ induce a $\mathfrak{A}_{2}$ Case 1 is settled.

Case $2 N\left(v_{j}\right) \cap\left\{x_{i}, y_{i}\right\}=\emptyset$ and $N\left(v_{k}\right) \cap\left\{x_{j}, y_{j}\right\}=\emptyset$.

Then $\left|N\left(v_{k}\right) \cap\left\{x_{i}, y_{i}\right\}\right|=1$, say $v_{k} \sim x_{i}$, and $\left|N\left(v_{i}\right) \cap\left\{x_{j}, y_{j}\right\}\right|=1$, say $v_{i} \sim x_{j}$. It follows that $x_{i} \notin\left\{x_{j}, y_{j}\right\}, x_{j} \notin\left\{x_{i}, y_{i}\right\}$.

Suppose $x_{i} \sim y_{j}$. Then $x_{i}, y_{j}, x_{j}, v_{i}, v_{k}$ induce a $C_{5}$ (if $x_{i} \not x_{j}$ ) or $x_{i}, x_{j}, y_{j}, v_{i}, v_{j}, v_{k}$ induce a $\mathfrak{A}_{12}$ (co-domino) (if $x_{i} \sim x_{j}$ ). Thus, $x_{i} \nsim y_{j}$. Likewise, $x_{j} \not y_{i}$. In particular, $y_{i} \neq y_{j}$, and $y_{i} \not y_{j}$ otherwise $y_{i}, y_{j}, v_{i}, v_{j}, v_{k}$ would induce a $\mathfrak{A}_{4}\left(K_{2} \cup K_{3}\right)$

Now, $v_{i}, v_{j}, v_{k}, x_{i}, y_{i}, x_{j}, y_{j}$ induce a $\mathfrak{A}_{5}$ (longhorn) (if $x_{i} \not x_{j}$ ) or a $\mathfrak{A}_{15}$ (otherwise). Case 2 is settled, completing the proof of Prop. 6.c. 


\subsection{The proof of Proposition 7}

Before we prove Prop. 77, we need some auxiliary results. The next three lemmas deal with the situation when

$$
\text { for every } c \in C: c \text { (1) } X \text { or } c \text { (1) } Y \text {. }
$$

Let $c_{1} \neq c_{2} \in C$ such that

$$
\begin{array}{ll}
c_{1} \text { (1) } X, & c_{1}(1) Y, \\
c_{2} \text { (1) } Y, & c_{2}(1) X .
\end{array}
$$

Lemma 13 Assume $C(1) X$ and $C(1) Y$. Let $u \in X$ and $v \in Y$ such that $c_{2} \not u$ and $c_{1} \nsim v$. If $u$ and $v$ are adjacent, then, for all adjacent vertices $x \in X \backslash\{u\}$ and $y \in Y \backslash\{v\}, c_{1} \not y$ or $c_{2} \nsim x$.

Proof: For contradiction, assume there are adjacent vertices $x \in X \backslash\{u\}, y \in Y \backslash\{v\}$ such that $c_{1} \sim y$ and $c_{2} \sim x$. Then $x \sim v$ or $y \sim u$, but not both (otherwise $c_{1}, c_{2}, u, v, x, y$ would induce a $\mathfrak{A}_{23}$ or a $\mathfrak{A}_{24}$ $\left(\overline{2 P_{3}}\right)$. By symmetry, we may assume

$$
x \sim v \quad \text { and } y \not u
$$

Claim $1 x$ (1) $C$

Proof: Assume $x \nsim c$ for some $c \in C \backslash\left\{c_{1}, c_{2}\right\}$. By (5), $c$ (1) $Y$. Then $u, v, c, c_{1}, y, x$ induce a $\mathfrak{A}_{26}$ $\overline{\left(P_{6}\right)}($ if $c \sim u)$, or $u, v, c, c_{1}, c_{2}, x, y$ induce a $\mathfrak{A}_{18}($ if $c \not u)$.

As $x \in B$, there exist adjacent vertices $x^{\prime}, y^{\prime} \in \bar{N}(x)$. As $x$ (1) $C$, we may assume that $x^{\prime} \in X \backslash\{x\}$, $y^{\prime} \in Y \backslash\{v, y\}$.

Suppose $y^{\prime} \sim u$. Then $x, y, c_{1}, c_{2}, u, y^{\prime}$ induce a $\overline{\mathfrak{A}_{23}}$ (if $y^{\prime} \nsim c_{1}$ ), or $u, v, c_{1}, c_{2}, x, y^{\prime}$ induce a $2 \mathfrak{A}_{14}$ (if $\left.y^{\prime} \sim c_{1}\right)$. Thus,

$$
y^{\prime} \nsim u \text {. }
$$

In particular, $x^{\prime} \neq u$.

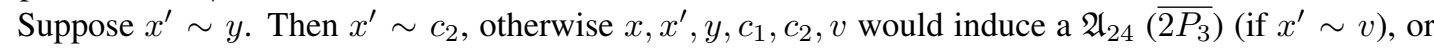
$x, x^{\prime}, y, c_{1}, c_{2}, u, v$ would induce a $\mathfrak{A}_{25}$ (if $x^{\prime} \nsim v$ ). Now, $x^{\prime}, y, c_{1}, c_{2}, u, v$ induce a $\mathfrak{A}_{23}$ (if $x^{\prime} \nsim v$ ), or $x, x^{\prime}, y, c_{1}, c_{2}, u, v$ induce a $\widehat{\mathfrak{A}_{18}}\left(\right.$ if $x^{\prime} \sim v$ ). Thus,

$$
x^{\prime} \nsim y .
$$

Therefore, $x^{\prime} \sim c_{2}$ or $y^{\prime} \sim c_{1}$ (otherwise $x, y, c_{1}, c_{2}, x^{\prime}, y^{\prime}$ would induce a $\mathfrak{A}_{23}$. Now, if $x^{\prime} \sim c_{2}$ and $y^{\prime} \sim c_{1}$ then $x^{\prime}, y^{\prime}, c_{1}, c_{2}, u, v$ induce a $\mathfrak{A}_{23}$ (if $x^{\prime} \not v$ ), or $x, y, x^{\prime}, y^{\prime}, c_{1}, c_{2}, u, v$ induce a $\mathfrak{A}_{44}\left(\right.$ if $x^{\prime} \sim v$ ). If $x^{\prime} \sim c_{2}$ and $y^{\prime} \nsim c_{1}$ then $x, x^{\prime}, y, y^{\prime}, c_{1}, c_{2}, u, v$ induce a $\mathfrak{A}_{40}$ (if $x^{\prime} \nsim v$ ), or a $\mathfrak{A}_{45}$ (if $x^{\prime} \sim v$ ). If $x^{\prime} \nsim c_{2}$ and $y^{\prime} \sim c_{1}$ then $x, x^{\prime}, y^{\prime}, c_{1}, c_{2}, v$ induce a $\overline{\mathfrak{A}_{26}\left(\overline{P_{6}}\right)}$ (if $x^{\prime} \sim v$ ), or $x, x^{\prime}, y^{\prime}, c_{1}, c_{2}, u, v$ induce a $\mathfrak{A}_{27}\left(\right.$ if $\left.x^{\prime} \nsim v\right)$.

Lemma 14 Assume $C(1) X$ and $C(1) Y$. Let $v_{1} v_{2} v_{3} v_{4}$, be an induced path in $B$ connecting $v_{1} \in X$ and $v_{4} \in Y$. If $c_{1} \not v_{4}$ and $c_{2} \not v_{1}$ then $c_{1} \not v_{2}$ or $c_{2} \nsim v_{3}$. 
Proof: For a contradiction, assume that $c_{1} \sim v_{2}$ and $c_{2} \sim v_{3}$. We will show that $v_{2}$ (1) $C$ and $v_{3}$ (1) $C$. As a first step we prove:

Claim $1 \quad v_{2}$ (1) $C$ or $v_{3}$ (1) $C$

Proof: Suppose $v_{2} \nsim c_{1}^{\prime}$ and $v_{3} \not c_{2}^{\prime}$ for some $c_{1}^{\prime}, c_{2}^{\prime} \in C \backslash\left\{c_{1}, c_{2}\right\}$. By [5], $c_{1}^{\prime}$ (1) $X$ and $c_{2}^{\prime}$ (1)

$Y$. In particular, $c_{1}^{\prime} \neq c_{2}^{\prime}$. Moreover, $c_{1}^{\prime} \sim v_{4}$ or $c_{2}^{\prime} \sim v_{1}$ (otherwise $c_{1}, c_{2}, c_{1}^{\prime}, c_{2}^{\prime}, v_{1}, v_{2}, v_{3}, v_{4}$ would induce a $\mathfrak{A}_{46}$, but not both (otherwise $c_{1}^{\prime}, c_{2}^{\prime}, v_{1}, v_{2}, v_{3}, v_{4}$ would induce a $\mathfrak{A}_{26}\left(\overline{P_{6}}\right)$. Now $v_{1}, v_{2}, v_{3}, v_{4}, c_{1}^{\prime}, c_{2}^{\prime}$, and $c_{1}$ (if $c_{1}^{\prime} \nsim v_{4}$ ) or $c_{2}$ (if $c_{2}^{\prime} \nsim v_{1}$ ) would induce a $\mathfrak{A}_{18}$.

Let, without loss of generality,

$$
v_{2} \text { (1) } C \text {. }
$$

As $v_{2} \in B$, there exist adjacent vertices $x, y \in \bar{N}\left(v_{2}\right)$. As $v_{2}(1) C$, we may assume that $x \in X \backslash\left\{v_{1}, v_{3}\right\}$, $y \in Y \backslash\left\{v_{2}\right\}$. Moreover,

$$
x \nsim v_{4}
$$

otherwise $v_{1}, v_{2}, v_{3}, v_{4}, c_{1}, c_{2}$ and $x$ would induce a $\widehat{\mathfrak{A}_{25}}\left(\right.$ if $c_{2} \nsim x$ ) or a $\mathfrak{A}_{28}\left(\right.$ if $c_{2} \sim x$ ). In particular, $y \neq v_{4}$.

Suppose $y \nsim v_{3}$. Then $y \nsim v_{1}$ otherwise $v_{1}, v_{2}, v_{3}, v_{4}, c_{1}, c_{2}$ and $y$ would induce a $\mathfrak{A}_{25}$ (if $c_{1} \nsim y$ ) or a $\mathfrak{A}_{28}$ (if $c_{1} \sim y$ ). Moreover, $c_{1} \sim y$ or $c_{2} \sim x$ otherwise $c_{1}, c_{2}, x, y, v_{2}, v_{3}$ would induce a $\mathfrak{A}_{23}$. But now $c_{1}, c_{2}, x, y, v_{1}, v_{2}, v_{3}, v_{4}$ induce a $\mathfrak{A}_{31}$ (if $c_{1} \sim y$ and $c_{2} \sim x$ ) or a $\mathfrak{A}_{30}$ (if $c_{1} \not y$ or $c_{2} \not x$ ). Thus,

$$
y \sim v_{3} .
$$

Hence also

$$
y \sim c_{1},
$$

otherwise $v_{1}, v_{2}, v_{3}, c_{1}, c_{2}, y$ would induce a $\overline{\mathfrak{A}_{24}\left(\overline{2 P_{3}}\right)}$ (if $\left.y \sim v_{1}\right)$, or $v_{1}, v_{2}, v_{3}, c_{1}, c_{2}, x, y$ would induce a $\mathfrak{A}_{25}$ (if $y \nsim v_{1}, x \nsim c_{2}$ ) or a $\mathfrak{A}_{28}$ (if $y \nsim v_{1}, x \sim c_{2}$ ).

We next show:

Claim $2 v_{3}$ (1) $C$

Proof: Suppose $v_{3} \nsim c_{2}^{\prime}$ for some $c_{2}^{\prime} \in C \backslash\left\{c_{1}, c_{2}\right\}$. By (5), $c_{2}^{\prime}$ (1) $Y$. By Lem. 13, $c_{2}^{\prime} \nsim v_{1}$ $\left(c_{2}:=c_{2}^{\prime}, u:=v_{3}, v:=v_{4}, x:=v_{1}, y:=v_{2}\right)$ and $c_{2}^{\prime} \not x\left(c_{2}:=c_{2}^{\prime}, u:=v_{3}, v:=v_{4}, x:=\right.$ $x, y:=y$ ). Moreover, $y \nsim v_{1}$ otherwise $v_{1}, v_{2}, v_{3}, v_{4}, c_{1}, c_{2}, c_{2}^{\prime}$ and $y$ would induce a $\mathfrak{A}_{46}$. Now $v_{1}, v_{2}, v_{3}, v_{4}, c_{1}, c_{2}, c_{2}^{\prime}, x$ and $y$ induce a $\mathfrak{A}_{47}\left(\right.$ if $c_{2} \not x$ ) or a $\mathfrak{A}_{48}\left(\right.$ if $c_{2} \sim x$ ). Claim 2 follows.

As $v_{3} \in B$, there exist adjacent vertices $x^{\prime}, y^{\prime} \in \bar{N}\left(v_{3}\right)$. As $v_{3}$ (1) $C$, we may assume that $y^{\prime} \in$ $Y \backslash\left\{y, v_{2}, v_{4}\right\}$ and $x^{\prime} \in X \backslash\left\{v_{3}\right\}$. Now, by symmetry, we obtain from (6), (7), and (8):

$$
y^{\prime} \nsim v_{1}, x^{\prime} \sim v_{2}, \quad \text { and } \quad x^{\prime} \sim c_{2} .
$$

In particular, $x^{\prime} \in X \backslash\left\{x, v_{1}, v_{3}\right\}$. Moreover,

$$
x \not y^{\prime}
$$

otherwise $c_{1}, c_{2}, v_{2}, v_{3}, x, y^{\prime}$ would induce a $\mathfrak{A}_{23}$ (if $x \nsim c_{2}$ and $y^{\prime} \nsim c_{1}$ ), or $c_{1}, c_{2}, v_{1}, v_{2}, v_{3}, v_{4}, x, y^{\prime}$ would induce a $\mathfrak{A}_{31}$ (if $x \sim c_{2}$ and $y^{\prime} \sim c_{1}$ ), or a $\mathfrak{A}_{30}$ (otherwise).

Suppose $y \sim x^{\prime}$. Then $y \nsim v_{1}$ (otherwise $v_{1}, v_{2}, v_{3}, v_{4}, c_{1}, c_{2}, y, x^{\prime}$ would induce a $\mathfrak{A}_{46}$ (if $x^{\prime} \sim v_{4}$ ), or $v_{1}, v_{2}, v_{3}, v_{4}, c_{1}, c_{2}, y, x^{\prime}, y^{\prime}$ would induce a $\mathfrak{A}_{47}\left(\right.$ if $\left.x^{\prime} \not v_{4}, y^{\prime} \not c_{1}\right)$ or a $\mathfrak{A}_{48}$ (if $\left.x^{\prime} \not v_{4}, y^{\prime} \sim c_{1}\right)$ ), and, 
by symmetry, $x^{\prime} \nsim v_{4}$. Now $v_{1}, v_{2}, v_{3}, v_{4}, c_{1}, c_{2}, x, y, x^{\prime}, y^{\prime}$ induce a $\mathfrak{A}_{49}$ or a $\mathfrak{A}_{50}$ (if $x \nsim c_{2}$ or $y^{\prime} \nsim c_{1}$ ) or a $\mathfrak{A}_{51}$ (if $x \sim c_{2}$ and $y^{\prime} \sim c_{1}$ ). Thus,

$$
y \nsim x^{\prime} .
$$

Furthermore,

$$
y \nsim v_{1}
$$

otherwise $v_{1}, v_{3}, v_{4}, c_{1}, c_{2}, y, x^{\prime}$ would induce a $\mathfrak{A}_{28}$ (if $x^{\prime} \sim v_{4}$ ), or $v_{1}, v_{3}, v_{4}, c_{1}, c_{2}, y, x^{\prime}, y^{\prime}$ would induce a $\mathfrak{A}_{30}\left(\right.$ if $x^{\prime} \nsim v_{4}, y^{\prime} \nsim c_{1}$ ) or a $\mathfrak{A}_{31}$ (if $x^{\prime} \nsim v_{4}, y^{\prime} \sim c_{1}$ ). By symmetry,

$$
x^{\prime} \nsim v_{4} \text {. }
$$

Suppose $x \not c_{2}$. Then $v_{3}, v_{4}, c_{1}, c_{2}, x, y, x^{\prime}, y^{\prime}$ induce a $\mathfrak{A}_{30}\left(\right.$ if $\left.y^{\prime} \nsim c_{1}\right)$ or a $\mathfrak{A}_{31}\left(\right.$ if $\left.y^{\prime} \sim c_{1}\right)$. Thus,

$$
x \sim c_{2},
$$

and by symmetry,

$$
y^{\prime} \sim c_{1} .
$$

Now $v_{1}, v_{2}, v_{3}, v_{4}, c_{1}, c_{2}, x, y, x^{\prime}, y^{\prime}$ induce a $\mathfrak{A}_{52}$

Lemma 15 Let $v_{1} v_{2} \cdots v_{2 k-1} v_{2 k}, k \geq 2$, be an induced path in $B$ connecting $v_{1} \in X$ and $v_{2 k} \in Y$. If $c_{1} \nsim v_{2 k}$ and $c_{2} \nsim v_{1}$ then $c_{1}$ (1) $\left\{v_{2 i} \mid i=1, \ldots, k\right\}$ or $c_{2}$ (1) $\left\{v_{2 j-1} \mid j=1, \ldots, k\right\}$.

Proof: The proof is by induction on $k$. In case $k=2$, Lem. 15 follows from Lem. 14

Let $k>2$. First, suppose $c_{1} \not v_{2 k-2}$. Then, by induction (applied to $v_{1} v_{2} \cdots v_{2 k-2}$ ),

$$
c_{1} \text { (0) }\left\{v_{2 i} \mid i=1, \ldots, k-1\right\} \text {, }
$$

or

$$
c_{2} \text { (1) }\left\{v_{2 j-1} \mid j=1, \ldots, k-1\right\} \text {. }
$$

As $c_{1} \nsim v_{2 k}$, we are done in case (9). Also, if $c_{2} \nsim v_{2 k-1}$ then we are done in case (10). So, let us assume that (9) does not hold, hence (10) does hold, and $c_{2} \sim v_{2 k-1}$. Let $c_{1} \sim v_{2 i}$ for some $i \in\{1, \ldots, k-2\}$. Now, if $i=k-2$ then $v_{2 k-4}, v_{2 k-3}, v_{2 k-2}, v_{2 k-1}, c_{1}, c_{2}$ induce a $\mathfrak{A}_{26}\left(\overline{P_{6}}\right)$ If $i<k-2, v_{2 i-1}, v_{2 i}$, $v_{2 k-3}, v_{2 k-2}, v_{2 k-1}, c_{1}, c_{2}$ induce a $\mathfrak{A}_{27}$ Thus, the case $c_{1} \not v_{2 k-2}$ is settled.

Now suppose $c_{1} \sim v_{2 k-2}$, and by symmetry, $c_{2} \sim v_{3}$. Then $c_{1} \sim v_{2}$ because otherwise $v_{1}, v_{2}$, $v_{2 k-2}, v_{2 k-1}, c_{1}, c_{2}$ would induce a $\mathfrak{A}_{23}$ (if $c_{2} \sim v_{2 k-1}$ ), or $v_{1}, v_{2}, v_{3}, v_{2 k-2}, v_{2 k-1}, c_{1}, c_{2}$ would induce a $\mathfrak{A}_{27}$ (if $c_{2} \nsim v_{2 k-1}$ ).

Now, if $c_{1} \nsim v_{4}$ then $v_{1}, v_{2}, v_{3}, v_{4}, v_{5}, c_{1}, c_{2}$ induce a $\mathfrak{A}_{25}$ (if $c_{2} \nsim v_{5}$ ) or a $\mathfrak{A}_{28}\left(\right.$ if $c_{2} \sim v_{5}$ ). If $c_{1} \nsim v_{2 i}$ for some $3 \leq i \leq k-1$, then by induction (applied to $v_{1} v_{2} \cdots v_{2 i}$ ), $c_{1} \nsim v_{2}$ or $c_{2} \nsim v_{3}$, a contradiction. Hence,

$$
c_{1} \text { (1) }\left\{v_{2 i} \mid i=1, \ldots, k-1\right\} \text {. }
$$

By symmetry we have

$$
c_{2} \text { (1) }\left\{v_{2 j-1} \mid j=2, \ldots, k\right\} .
$$

But then $c_{1}, c_{2}$ and $v_{1}, \ldots, v_{2 k}$, induce a $\mathfrak{\mathscr { A }}_{3}$. 
Lemma 16 (Prop.7) For every non-trivial connected component $B^{\prime}=\left(X \cup Y, E^{\prime}\right)$ of $G[B], X$ (1) $C$ or $Y$ (1) $C$.

\section{Proof:}

CLAIM 1 For every induced path $v_{1} v_{2} \cdots v_{2 k-1} v_{2 k}, k \geq 3$, in $B$ and for every vertex $v \in C: v \sim v_{1}$ or $v \sim v_{2 k}$

Proof: The proof is by induction on $k$. First, let $k=3$. If $v \not v_{1}$ and $v \nsim v_{6}$ then, as $v \in C$ and because of Prop. 6.a $v \sim v_{2}, v \sim v_{5}$. Discussing the adjacencies between $v$ and $v_{3}, v_{4}$ we conclude that there is an induced $C_{5}$, or a $\mathfrak{A}_{11}$ (antenna), or a $\mathfrak{A}_{2}$ Thus, $v \sim v_{1}$ or $v \sim v_{6}$, and Claim 1 is proved for $k=3$.

Next, let $k \geq 4$, and assume that $v \nsim v_{1}$ and $v \nsim v_{2 k}$. By Prop. 6.a $v \sim v_{2}, v \sim v_{2 k-1}$. Let $i$, $3 \leq i \leq 2 k-2$, be the smallest index such that $v \not v_{i}$; such an $i$ exists otherwise $v, v_{1}, \ldots, v_{2 k}$ would induce a $\mathfrak{A}_{2}$. Note that, by the choice of $i, v \sim v_{j}, j=2,3, \ldots i-1$, and by Prop. 6.a. $v \sim v_{i+1}$. Moreover, $i$ must be odd otherwise $v, v_{1}, v_{2}, \ldots, v_{i}$ would induce a $\mathfrak{A}_{2}$ (if $i \geq 6$ ) or $v, v_{1}, v_{2}, v_{3}, v_{4}, v_{5}$ would induce a $\mathfrak{A}_{11}$ (antenna) (if $i=4$ ).

Now, if $i \leq 2 k-5$ then, by applying the induction hypothesis for the path $v_{i}, v_{i+1} \cdots v_{2 k}, v \sim v_{i}$ or $v \sim v_{2 k}$, a contradiction. Thus, $i \geq 2 k-3$, hence $i=2 k-3$. Then $v, v_{2 k-4}, v_{2 k-3}, v_{2 k-2}$, $v_{2 k-1}, v_{2 k}$ induce a $\mathfrak{A}_{11}$ (antenna)

Let $B^{\prime}=\left(X \cup Y, E\left(B^{\prime}\right)\right)$ be a nontrivial connected component of $B$, and assume to the contrary that $X(1) C$ and $Y(1) C$. We distinguish two cases.

Case 1: There exists a vertex $c \in C$ non-adjacent to a vertex $a \in X$ and non-adjacent to a vertex $b \in Y$.

As $c \in C$ and because of Prop. 6.a $a \nsim b$. Hence, by Claim 1. every shortest path in $B^{\prime}$ connecting $a$ and $b$ must consist of exactly four vertices (including $a, b$ ); let $v_{1} v_{2} v_{3} v_{4}$ with $v_{1}=a$ and $v_{4}=b$ be a shortest path in $B^{\prime}$.

As $c \in C$ and by Prop. 6.a. $c \sim v_{2}, c \sim v_{3}$. We will show that $v_{2}$ (1) $C$ and $v_{3}$ (1) $C$. As a first step, we prove $v_{2}$ (1) $C$ or $v_{3}$ (1) $C$ :CLAIM $2 v_{2}$ (1) $C$ or $v_{3}$ (1) $C$.

Proof: Suppose $v_{2} \nsim c^{\prime}$ and $v_{3} \nsim c^{\prime \prime}$ for some $c^{\prime}, c^{\prime \prime} \in C \backslash\{c\}$. Then $c^{\prime}$ is adjacent to $v_{1}, v_{3}$ and $c^{\prime \prime}$ is adjacent to $v_{2}, v_{4}$ because of Prop. 6.a In particular, $c^{\prime} \neq c^{\prime \prime}$. Moreover, $c^{\prime} \sim v_{4}$ or $c^{\prime \prime} \sim v_{1}$ (otherwise $c, c^{\prime}, c^{\prime \prime}, v_{1}, v_{2}, v_{3}, v_{4}$ would induce a $\mathfrak{A}_{20}$, but not both (otherwise $v_{1}, v_{2}, v_{3}, v_{4}, c^{\prime}, c^{\prime \prime}$ would induce a $\mathfrak{A}_{26}\left(\overline{P_{6}}\right)$. Now, $c, c^{\prime}, c^{\prime \prime}, v_{1}, v_{2}, v_{3}, v_{4}$ induce a $\mathfrak{A}_{10}$, regardless whether $c^{\prime} \nsim v_{4}$ or $c^{\prime \prime} \nsim v_{1}$, and Claim 2 follows.

By symmetry, we may assume that

$$
v_{2} \text { (1) } C \text {. }
$$

As $v_{2} \in B$, there exist adjacent vertices $x, y \in \bar{N}\left(v_{2}\right)$. As $v_{2}$ (1) $C$, we may assume that $x \in X \backslash\left\{v_{1}, v_{3}\right\}$, $y \in Y \backslash\left\{v_{2}\right\}$.

Suppose that $x \sim v_{4}$. Then $c \sim x$ (as $c \in C$ ), and $c, v_{1}, v_{2}, v_{3}, v_{4}, x$ induce a $\mathfrak{A}_{11}$ (antenna). Thus,

$$
x \nsim v_{4} .
$$

In particular, $y \neq v_{4}$. Moreover,

$$
y \sim c
$$


otherwise $c \sim x$ (by Prop. 6.a) and therefore $y \nsim v_{1}$ (else $y, v_{1}, v_{2}, c, x$ would induce a $C_{5}$ ), implying that either $v_{1}, v_{2}, v_{3}, c, x, y$ induce a $\mathfrak{A}_{11}$ (antenna) (if $y \sim v_{3}$ ), or $v_{1}, v_{2}, v_{3}, v_{4}, c, x, y$ induce a $\mathfrak{A}_{6}$ (eiffeltower) Furthermore,

$$
y \sim v_{3},
$$

otherwise $v_{1}, v_{2}, v_{3}, v_{4}, c, y$ would induce a $\mathfrak{A}_{11}$ (antenna) (if $y \sim v_{1}$ ), or $v_{1}, v_{2}, v_{3}, v_{4}, c, x, y$ would induce a $\mathfrak{A}_{6}$ (eiffeltower) (if $y \nsim v_{1}$ and $x \not c$ ), or a $\mathfrak{A}_{7}$ (if $y \not v_{1}$ and $x \sim c$ ).

We next show:

Claim 3 v $v_{3}$ (1) $C$

Proof: Suppose $v_{3} \nsim c^{\prime}$ for some $c^{\prime} \in C \backslash\{c\}$. Then by Prop. 6.a. $c^{\prime} \sim v_{4}$ and $c^{\prime} \sim y$. Moreover, $y \nsim v_{1}$ otherwise $v_{1}, v_{2}, v_{3}, v_{4}, c, c^{\prime}, y$ would induce a $\mathfrak{A}_{20}$ (if $c^{\prime} \nsim v_{1}$ ) or a $\mathfrak{A}_{10}\left(\right.$ if $c^{\prime} \sim v_{1}$ ). Now, $v_{1}, v_{2}, v_{3}, v_{4}, c, c^{\prime}, x$ and $y$ induce a forbidden subgraph $H$ as follows:

- $c^{\prime} \nsim v_{1}, c^{\prime} \nsim x, c \nsim x: H$ is a $\mathfrak{A}_{36} \bullet c^{\prime} \sim v_{1}, c^{\prime} \nsim x, c \nsim x: H$ is a $\mathfrak{A}_{38}$

- $c^{\prime} \nsim v_{1}, c^{\prime} \sim x, c \neq x: H$ is a $\mathfrak{A}_{38} \bullet c^{\prime} \sim v_{1}, c^{\prime} \sim x, c \nsim x: H$ is a $\mathfrak{A}_{39}$

- $c^{\prime} \nsim v_{1}, c^{\prime} \nsim x, c \sim x: H$ is a $\mathfrak{A}_{37} \bullet c^{\prime} \sim v_{1}, c^{\prime} \nsim x, c \sim x: H$ is a $\mathfrak{A}_{42}$.

- $c^{\prime} \nsim v_{1}, c^{\prime} \sim x, c \sim x: H$ is a $\mathfrak{A}_{41} \bullet c^{\prime} \sim v_{1}, c^{\prime} \sim x, c \sim x: H$ is a $\mathfrak{A}_{43}$

Claim 3 follows.

As $v_{3} \in B$, there exist adjacent vertices $x^{\prime}, y^{\prime} \in \bar{N}\left(v_{3}\right)$. As $v_{3}(1) C$, we may assume that $x^{\prime} \in X \backslash\left\{v_{3}\right\}$, $y^{\prime} \in Y \backslash\left\{v_{2}, v_{4}\right\}$. By symmetry, we obtain from (11), 112, and (13):

$$
y^{\prime} \nsim v_{1}, x^{\prime} \sim c, \text { and } x^{\prime} \sim v_{2} .
$$

In particular, $x^{\prime} \neq v_{1}$, and $x, y, x^{\prime}, y^{\prime}$ are pairwise distinct.

Suppose $x \sim y^{\prime}$. Then $c$ is adjacent to precisely one of $x, y^{\prime}$ : otherwise either $v_{1}, v_{2}, v_{3}, v_{4}, c, x, y^{\prime}$ would induce a $\mathfrak{A}_{7}$ (if $\left.c \sim x, y^{\prime}\right)$, or $v_{2}, v_{3}, c, x, y^{\prime}$ would induce a $\mathfrak{A}_{4}\left(K_{2} \cup K_{3}\right)$ (if $\left.c \not x, y^{\prime}\right)$. By symmetry we may assume that $c \nsim x$ and $c \sim y^{\prime}$. Then $v_{3}, v_{4}, c, x, y, y^{\prime}$ induce a $\mathfrak{A}_{11}$ (antenna). Thus,

$$
x \nsim y^{\prime} .
$$

Suppose $y \sim x^{\prime}$. Then $x^{\prime} \sim v_{4}$ or $y \sim v_{1}$ (otherwise $v_{1}, v_{2}, v_{3}, v_{4}, c, x, y, x^{\prime}, y^{\prime}$ would induce a $\mathfrak{A}_{32}$. or a $\mathfrak{A}_{33}$, or a $\mathfrak{A}_{34}$, but not both (otherwise $v_{1}, v_{2}, v_{3}, v_{4}, c, y, x^{\prime}$ would induce a $\mathfrak{A}_{20}$ ). By symmetry we may assume that $x^{\prime} \nsim v_{4}$ and $y \sim v_{1}$. Then $v_{1}, v_{2}, v_{3}, v_{4}, c, y, x^{\prime}, y^{\prime}$ induce a $\mathfrak{A}_{36}$ (if $c \nsim y^{\prime}$ ), or a $\mathfrak{A}_{37}$ (if $\left.c \sim y^{\prime}\right)$. Thus,

$$
y \not x^{\prime} .
$$

Then $c \sim x$ or $c \sim y^{\prime}$ (otherwise $v_{3}, v_{4}, c, x, y, x^{\prime}$ would induce a $\mathfrak{A}_{11}$ (antenna) (if $x^{\prime} \sim v_{4}$ ), or $v_{3}, v_{4}, c, x, y, x^{\prime}, y^{\prime}$ would induce a $\mathfrak{A}_{6}$ (eiffeltower) (if $\left.x^{\prime} \nsim v_{4}\right)$ ), but not both (otherwise $v_{1}, v_{2}, v_{3}, v_{4}, c$, $x, y, x^{\prime}, y^{\prime}$ would induce a $\mathfrak{A}_{35}$ (if $y \nsim v_{1}, x^{\prime} \nsim v_{4}$ ), or $v_{1}, v_{2}, v_{4}, c, y, x^{\prime}$ would induce a $\mathfrak{A}_{11}$ (antenna) (if $y \sim v_{1}, x^{\prime} \sim v_{4}$ ), or $v_{1}, v_{3}, v_{4}, c, y, x^{\prime}, y^{\prime}$ would induce a $\mathfrak{A}_{7}$ (if $y \sim v_{1}, x^{\prime} \nsim v_{4}$ ); the case $y \nsim v_{1}$, $x^{\prime} \sim v_{4}$ is symmetrical to the last one). By symmetry we may assume that $c \sim x$ and $c \nsim y^{\prime}$. But then $v_{1}, v_{2}, c, y, x^{\prime}, y^{\prime}$ induce a $\mathfrak{A}_{11}$ (antenna) (if $\left.y \sim v_{1}\right)$, or $v_{1}, v_{2}, c, x, y, x^{\prime}, y^{\prime}$ induce a $\mathfrak{A}_{7}$ (if $y \nsim v_{1}$ ).

Case 1 is settled. 
Case 2: For every vertex $c \in C, c$ (1) $X$ or $c$ (1) $Y$.

In this case, since it is assumed that $C(\mathbb{1}) X$ and $C(1) Y$, there exist vertices $c_{1} \neq c_{2} \in C$ such that

$$
c_{1} \text { (1) } X, c_{1}(1) Y \text { and } c_{2} \text { (1) } X, c_{2} \text { (1) } Y \text {. }
$$

Observe that the prerequisites for Lem. 13, 14, 15, hold. We distinguish two subcases.

Case 2.1: For all $a \in X, b \in Y$, if $c_{2} \not a$ and $c_{1} \not b$ then $a \sim b$.

Since $c_{1}, c_{2} \in C$, there exist a neighbour $x \in X$ of $c_{2}$ and a neighbour $y \in Y$ of $c_{1}$. By Lem. 13 $x \not y$. Consider an induced path $\left(x=v_{1}, v_{2}, \cdots, v_{2 k-1}, v_{2 k}=y\right)$ in $B$ connecting $x$ and $y, k \geq 2$. By Lem. 13 again, $c_{1} \nsim v_{2}$ and $c_{2} \nsim v_{2 k-1}$. As we are in Case 2.1, $v_{2} \sim v_{2 k-1}$, hence $k=2$. Now $v_{1}, v_{2}, v_{3}, v_{4}, c_{1}, c_{2}$ induce a $\mathfrak{A}_{26}\left(\overline{P_{6}}\right)$

Case 2.1 is settled.

Case 2.2: $\quad$ There exist $a \in X, b \in Y$ such that $c_{2} \nsim a, c_{1} \nsim b$, and $a \neq b$.

Let $a \in X$ and $b \in Y$ be two such vertices, and consider an induced path $\left(a=v_{1}, v_{2}, \cdots, v_{2 k-1}, v_{2 k}=\right.$ $b$ ) in $B$ connecting $a$ and $b$. As $a \not b, k \geq 2$. By Lem. 15, we may assume without loss of generality,

$$
c_{1}(0)\left\{v_{2 i} \mid 1 \leq i \leq k\right\}
$$

As $c_{1} \in C, c_{1}$ has a neighbour $y \in Y \backslash\left\{v_{2}, v_{4}, \ldots, v_{2 k}\right\}$.

Case 2.2.1: $\quad c_{2}$ (1) $\left\{v_{2 j-1} \mid 1 \leq j \leq k\right\}$.

As $c_{2} \in C, c_{2}$ has a neighbour $x \in X \backslash\left\{v_{1}, v_{3}, \ldots, v_{2 k-1}\right\}$. By Lem. 13 .

$$
x \nsim y \text {. }
$$

CLAIM $4 x$ (0) $\left\{v_{2 i} \mid 1 \leq i \leq k\right\}$ or $y$ (0) $\left\{v_{2 j-1} \mid 1 \leq j \leq k\right\}$

PROOF: Suppose $x \sim v_{2 i}$ for some $1 \leq i \leq k$. Then $y \not v_{2 i-1}$, and if $i<k, y \neq v_{2 i+1}$, otherwise $c_{1}, c_{2}, x, y, v_{2 i}$, and $v_{2 i-1}$ (and if $\left.i<k, v_{2 i+1}\right)$ would induce a $\mathfrak{A}_{26}\left(\overline{P_{6}}\right)$, hence if $v \in\left\{v_{2 j-1}\right.$ $1 \leq j \leq k\}$ were a neighbour of $y$, then $c_{1}, c_{2}, x, y, v, v_{2 i}, v_{2 i-1}$ would induce a $\mathfrak{A}_{27}$. Thus, $y$ (1) $\left\{v_{2 j-1} \mid 1 \leq j \leq k\right\}$. By symmetry, if $y$ (D) $\left\{v_{2 j-1} \mid 1 \leq j \leq k\right\}$, then $x$ (1) $\left\{v_{2 i} \mid 1 \leq i \leq k\right\}$.

By symmetry we may assume that

$$
x \text { (1) }\left\{v_{2 i} \mid 1 \leq i \leq k\right\} \text {. }
$$

As $B$ is connected, $x$ therefore has a neighbour $y^{\prime} \in Y \backslash\left\{y, v_{2}, v_{4}, \ldots, v_{2 k}\right\}$. By Lem. 13 (applied to $u=v_{1}, v=v_{2}, x$ and $\left.y^{\prime}\right)$,

$$
c_{1} \not y^{\prime}
$$

Moreover,

$$
y \text { (1) }\left\{v_{2 j-1} \mid 1 \leq j \leq k\right\}:
$$

For, if $y \sim v_{2 j-1}$ for some $1 \leq j \leq k$, then $y^{\prime} \not v_{2 j-1}$ otherwise $c_{1}, c_{2}, x, y, v_{2 j-1}, y^{\prime}$ would induce a $\mathfrak{A}_{26}\left(\overline{P_{6}}\right)$. Now $c_{1}, c_{2}, x, y, v_{2 j-1}, y^{\prime}$ and $v_{2 j}$ induce a $\mathfrak{A}_{27}$. 
As $B$ is connected, $y$ must have a neighbour $x^{\prime} \in X \backslash\left\{x, v_{1}, v_{3}, \ldots, v_{2 k-1}\right\}$. By Lem. 13 again,

$$
c_{2} \not x^{\prime}
$$

Hence

$$
x^{\prime} \nsim y^{\prime}
$$

otherwise $c_{1}, c_{2}, x, y, x^{\prime}, y^{\prime}$ would induce a $\overline{\mathfrak{A}_{26}\left(\overline{P_{6}}\right)}$. Now $c_{1}, c_{2}, x, y, x^{\prime}, y^{\prime}$, and $v_{1}$ (if $\left.y^{\prime} \sim v_{1}\right)$ or $v_{2}$ (if $x^{\prime} \sim v_{2}$ ) induce a $\mathfrak{A}_{27}$ or $c_{1}, c_{2}, x, y, x^{\prime}, y^{\prime}, v_{1}, v_{2}$ induce a $\mathfrak{A}_{19}$ (if $y^{\prime} \nsim v_{1}$ and $x^{\prime} \nsim v_{2}$ ).

Case 2.2.1 is settled.

Case 2.2.2: $\quad c_{2} \sim v_{2 j-1}$ for some $1 \leq j \leq k$.

Fix such an index $j$. Since $c_{2} \nsim v_{1}, j>1$. By Lem. 13 (applied to $u=v_{1}, v=v_{2}, x=v_{2 j-1}$ and $y$ ),

$$
y \not v_{2 j-1} \text {. }
$$

Suppose $y \sim v_{2 j^{\prime}-1}$ for some $1 \leq j^{\prime} \leq k, j^{\prime} \neq j$. Then, by Lem. 13 again,

$$
c_{2} \not v_{2 j^{\prime}-1}
$$

If $j^{\prime}=j-1$ then $c_{1}, c_{2}, y, v_{2 j-1}, v_{2 j-2}, v_{2} j-3$ induce a $\overline{\mathfrak{A}_{26}\left(\overline{P_{6}}\right)}$. If $j^{\prime}=j+1$ (implying $j<k$ ) then $c_{1}, c_{2}, y, v_{2 j-1}, v_{2 j}, v_{2 j+1}$ induce a $\overline{\mathfrak{A}_{26}\left(\overline{P_{6}}\right)}$ If $j^{\prime} \neq j \pm 1, c_{1}, c_{2}, y, v_{2 j-1}, v_{2 j}, v_{2 j^{\prime}-1}, v_{2 j^{\prime}}$ induce a $\mathfrak{A}_{27}$. Thus,

$$
y \text { (1) }\left\{v_{1}, v_{3}, \ldots, v_{2 k-1}\right\} \text {. }
$$

As $B$ is connected, $y$ has a neighbour $x \in X \backslash\left\{v_{1}, v_{3}, \ldots, v_{2 k-1}\right\}$. By Lem.13 again,

$$
c_{2} \not x
$$

Now, if $x \sim v_{2 j-2}$ then $c_{1}, c_{2}, x, y, v_{2 j-2}, v_{2 j-3}$ induce a $\overline{\mathfrak{A}_{26}\left(\overline{P_{6}}\right)}$ and if $x \not v_{2 j-2}$ then $c_{1}, c_{2}, x, y$, $v_{2 j-1}, v_{2 j-2}, v_{2 j-3}$ induce a $\mathfrak{A}_{27}$

Case 2.2.2, hence Case 2, is settled, and the proof of Prop.7 is complete.

\subsection{The proof of Proposition 8}

Let $v \in V \backslash(B \cup C)$, that is, $N(v)$ induces a split graph and $\bar{N}(v)$ is independent. Assume that $G-v$ is probe split and let $(C, S, I)$ be a probe split partition of $G-v$. Create subpartitions of $S$ and $I$ as:

$$
\begin{aligned}
& S_{1}=S \cap N(v), \\
& S_{2}=S \backslash S_{1}, \\
& I_{1}=I \cap N(v), \\
& I_{2}=I \backslash I_{1} .
\end{aligned}
$$

As $\bar{N}(v)$ is an independent set, $S_{2}$ (0) $I_{2}$ and $|C \cap \bar{N}(v)| \leq 1$. Moreover, $C$ (1) $S_{2}$ and $S_{2} \subseteq \bar{N}(v)$ implies that if $S_{2} \neq \emptyset$, then $|C \cap \bar{N}(v)|=0$, that is $v$ (1) $C$.

Lemma 17 If $S_{1}$ (1) $I_{1}$ then $G$ is probe split. 
Proof: We split the proof into two cases.

1. $v$ (1) $C$. We may assume $S_{1}(0) I_{2}$ (otherwise $\left(C, S_{2} \cup\{v\}, I \cup S_{1}\right)$ is a valid probe split partition of $G), I_{1}\left(S_{2}\right.$ (otherwise $\left(C \cup\{v\}, S_{1}, I \cup S_{2}\right)$ is a valid probe split partition), and $C \neq \emptyset$ (otherwise $\left(\{v\}, S_{1} \cup I_{1}, S_{2} \cup I_{2}\right)$ is a valid probe split partition). In particular $I_{1}, I_{2}, S_{1}, S_{2}$ all are non-empty. Define

$$
\begin{aligned}
& I_{21}=I_{2} \cap N\left(S_{1}\right), \\
& I_{22}=I_{2} \backslash I_{21}, \\
& I_{11}=I_{1} \cap N\left(S_{2}\right), \\
& I_{12}=I_{1} \backslash I_{11} .
\end{aligned}
$$

Note that $I_{21}$ and $I_{11}$ are non-empty. We show that $I_{21}$ (1) $C$ or $I_{11}$ (1) $C$. Let $i_{11} \in I_{11}$ with a neighbour $s_{2} \in S_{2}$ and a non-neighbour $c \in C$ and let $i_{21} \in I_{21}$ with a neighbour $s_{1} \in S_{1}$ and a non-neighbour $c^{\prime} \in C$. If $c^{\prime}=c$ then $v c i_{11} i_{21} s_{1} s_{2}$ induce a $\mathfrak{A}_{11}$ (antenna) and if $c \neq c^{\prime}$ then $v c c^{\prime} i_{11} i_{21} s_{1} s_{2}$ induce a $\mathfrak{A}_{28}$. Thus either $I_{21}$ (1) $C$ or $I_{11}$ (1) $C$. If $I_{21}$ (1) $C$, then $\left(C,\{v\} \cup S_{2} \cup\right.$ $\left.I_{21}, I_{1} \cup I_{22} \cup S_{1}\right)$ is a valid probe split partition and if $I_{11}$ (1) $C$ then $\left(C \cup\{v\}, S_{1} \cup I_{11}, I_{12} \cup S_{2} \cup I_{2}\right)$ is a valid probe split partition.

2. $v$ (1) $C$. Because $\bar{N}(v)$ is independent, $v$ is non-adjacent to precisely one vertex $c$ of $C$. Then, for the same reason, $S_{2}=\emptyset$ and $c$ (0) $I_{2}$. Define

$$
\begin{aligned}
& I_{11}=I_{1} \cap N(c), \\
& I_{12}=I_{1} \backslash I_{11} .
\end{aligned}
$$

We may assume $I_{11}$ to be non-empty, because otherwise $\left(C-c+v, S_{1}, I+c\right)$ would be a valid probe split partition. We may also assume that $I_{11}(1)(C-c)$ because otherwise $\left(C-c+v, I_{11} \cup\right.$ $S_{1}, I_{2} \cup I_{12} \cup\{c\}$ ) would be a valid probe split partition. Let $i_{11} \in I_{11}$ and $c_{11} \in C-c$ such that $i_{11} c_{11} \notin E$ (note that $i_{11} c \in E$ ). Partition $I_{2}$ as:

$$
\begin{aligned}
I_{21} & =\left\{x \in I_{2}|| N(x) \cap S_{1} \mid \geq 2\right\}, \\
A & =\left\{x \in I_{2}|| N(x) \cap S_{1} \mid=1 \wedge x(1)(C-c)\right\}, \\
B & =\left\{x \in I_{2}|| N(x) \cap S_{1} \mid=1 \wedge x(1)(C-c)\right\}, \\
R & =I_{2} \backslash\left(I_{21} \cup A \cup B\right) .
\end{aligned}
$$

Note that by this definition $R$ (0) $S_{1}$ and $A$ (1) $(C-c)$. We first show that

$$
I_{21}(1)(C-c) \text {. }
$$

Let $x \in I_{21}$ with two neighbours $u, u^{\prime} \in S_{1}$ and a non-neighbour $c_{x} \in C-c$. If $i_{11} c_{x} \notin E$ then $v u u^{\prime} c x i_{11} c_{x}$ induces a $\mathfrak{2}_{20}$, if $x c_{11} \notin E$ then $v u u^{\prime} c x i_{11} c_{11}$ induces a $\mathfrak{A}_{20}$, and if both are edges then $v u u^{\prime} c x c_{x} i_{11} c_{11}$ induces a $\mathfrak{A}_{46}$ This proves (14).

We now may assume that $B \neq \emptyset$ because otherwise $\left(C-c, I_{21} \cup A \cup\{c, v\}, I_{1} \cup R \cup S_{1}\right)$ is a valid probe split partition. We next show that precisely one vertex $s_{B} \in S_{1}$ exists such that $s_{B}$ is the unique neighbour in $S_{1}$ of every vertex in $B$, that is

$$
N(B) \cap S_{1}=\left\{s_{B}\right\} .
$$


Assume there are $b_{1}, b_{2} \in B$ with unique neighbour $s_{1}, s_{2} \in S_{1}$, respectively $\left(b_{1} \neq b_{2}, s_{1} \neq s_{2}\right)$. Then $c_{11} b_{1}$ and $c_{11} b_{2}$ are both edges, because otherwise $b_{1} s_{1} b_{2} s_{2} v c i_{11} c_{11}$ would induce a $\mathfrak{A}_{37}$ or a $\mathfrak{A}_{36}$ According to the definition of $B$, a vertex $c_{1} \in C-c$ exists that is a non-neighbour of $b_{1} . \quad c_{1} i_{11} \in E$, because otherwise we again have a $\mathfrak{A}_{37}$ or a $\mathfrak{A}_{36}\left(b_{1} s_{1} b_{2} s_{2} v c i_{11} c_{1}\right)$. But now $b_{1} s_{1} b_{2} s_{2} v c i_{11} c_{11} c_{1}$ induces a $\mathfrak{A}_{47}$ (if $c_{1} b_{2} \notin E$ ) or a $\mathfrak{A}_{48}$ (if $c_{1} b_{2} \in E$ ). This proves $s_{1}=s_{2}$ and thus 15 .

Finally we show that

$$
s_{B}(1)\left(I_{21} \cup A\right) \text {. }
$$

Let $x \in I_{21} \cup A$ be a non-neighbour of $s_{B}$ and let $s_{1} \in S_{1}$ be a neighbour of $x$. Let $b \in B$ with non-neighbour $c_{b} \in C-c$. By (14) and the definition of $A, x$ (1) $(C-c)$. Both $i_{11} c_{b}$ and $c_{11} b$ are edges because otherwise $i_{11} c v s_{1} s_{B} b x c_{b}$, and $i_{11} c v s_{1} s_{B} b x c_{11}$, respectively, induce a $\mathfrak{A}_{36}$. But then $i_{11} c v s_{1} s_{B} b x c_{11} c_{b}$ induce a $\mathfrak{2}_{48}$ (16) follows.

We claim that $(Q, U, J)$ with

$$
\begin{aligned}
Q & =C-c+s_{B}, \\
U & =I_{21} \cup A \cup\{c, v\}, \\
J & =I_{1} \cup R \cup B \cup\left(S_{1}-s_{B}\right)
\end{aligned}
$$

is a valid probe split partition: Independence of $U$ and $J$ is clear by definition of $A, B, R$ and $s_{B}$ and $Q$ (1) $U$ follows from (14) and (16).

We turn to the case when $S_{1}\left(\right.$ (b) $I_{1}$. Let $x \in S_{1}, y \in I_{1}$ with $x y \in E$ such that $\left|N(x) \cap I_{1}\right|+\left|N(y) \cap S_{1}\right|$ is maximum. Partition $I_{1}-y$ and $S_{1}-x$ as:

$$
\begin{aligned}
& I_{11}=\left(I_{1}-y\right) \cap N(x), \\
& I_{12}=\left(I_{1}-y\right) \backslash I_{11}, \\
& S_{11}=\left(S_{1}-x\right) \cap N(y), \\
& S_{12}=\left(S_{1}-x\right) \backslash S_{11} .
\end{aligned}
$$

For some of the following lemmas it is important to recall that $G[N(v)]$ is assumed to be a split graph and therefore $\left(2 K_{2}, C_{4}, C_{5}\right)$-free.

Lemma $18\left(S_{11} \cup S_{12}\right)$ (9) $\left(I_{11} \cup I_{12}\right)$

Proof: First, there cannot be an edge between $S_{11}$ and $I_{11}$ or between $S_{12}$ and $I_{12}$, because together with $x, y$ this edge would induce a $C_{4}$ or $2 K_{2}$, respectively, in $N(v)$. Secondly, suppose an edge $x^{\prime} y^{\prime}$ exists, with $x^{\prime} \in S_{11}, y^{\prime} \in I_{12}$. Then $I_{11}=\emptyset$, because otherwise $x, x^{\prime}, y^{\prime}$ and a vertex from $I_{11}$ would induce a $2 K_{2}$ in $N(v)$, which implies that $\left|N\left(x^{\prime}\right) \cap I_{1}\right|+\left|N(y) \cap S_{1}\right|>\left|N(x) \cap I_{1}\right|+\left|N(y) \cap S_{1}\right|$, contradicting the choice of $x, y$. Similarly, there is no edge between $I_{11}$ and $S_{12}$.

Lemma 19 If $S_{11} \neq \emptyset$ then $y(1)(C \cap N(v))$. 
Proof: If $y$ is non-adjacent to some $c \in(C \cap N(v))$ then $x, y, c$ and a vertex from $S_{11}$ induce a $C_{4}$ in $N(v)$.

Lemma 20 Either $S_{2}$ (0) $I_{11}$ or $I_{2}$ (0) $S_{11}$.

Proof: Assume there are edges $s_{2} i_{11}$ and $i_{2} s_{11}$ with $s_{2} \in S_{2}, i_{11} \in I_{11}, i_{2} \in I_{2}, s_{11} \in S_{11}$. If $x i_{2} \in E$ or $y s_{2} \in E$ then $v s_{2} i_{2} s_{11} i_{11}$ together with $x$ or $y$, respectively, induce a $\mathfrak{A}_{11}$ (antenna) , if neither are edges then $v s_{2} i_{2} s_{11} i_{11} x y$ induce a $\mathfrak{A}_{2}$

Lemma 21 a) Either $S_{2}$ (0) $I_{12}$ or $S_{2}$ (0) $\left(I_{11}+y\right)$ or $I_{2}$ (0) $\left(S_{11}+x\right)$.

b) Either $I_{2}$ (1) $S_{12}$ or $I_{2}$ (1) $\left(S_{11}+x\right)$ or $S_{2}$ (1) $\left(I_{11}+y\right)$.

Proof: We prove the first statement; the second is symmetrical. Assume there are edges $s_{2} i_{12}, s_{2}^{\prime} z$ and $i_{2} t$ with $s_{2}, s_{2}^{\prime} \in S_{2}, i_{12} \in I_{12}, i_{2} \in I_{2}, z \in I_{11}+y, t \in S_{11}+x$. By Lem.20, $z=y$ or $t=x$, implying that $z$ and $t$ are adjacent. If $z s_{2} \in E$ or $i_{12} s_{2}^{\prime} \in E$ then $v z t i_{2}$ together with $s_{2}$ or $s_{2}^{\prime}$, respectively, induces a $\mathfrak{A}_{11}$ (antenna), if neither are edges then $v z t i_{12} i_{2} s_{2} s_{2}^{\prime}$ induces a $\mathfrak{A}_{6}$ (eiffeltower)

Lemma 22 If $I_{2}$ (b) $S_{1}$ then $\left(I_{12} \cap N\left(S_{2}\right)\right)$ (1) $C$.

Proof: Seeking a contradiction, assume $i_{12} \in I_{12} \cap N\left(S_{2}\right)$ is non-adjacent to $c \in C$. Let $s_{2} \in S_{2}$ be a neighbour of $i_{12}$. We distinguish two cases matching the partition of $S_{1}$.

1. $I_{2}$ ( $\left(S_{11}+x\right)$

Consider an edge $i_{2} z$ with $i_{2} \in I_{2}, z \in S_{11}+x$. Then $z$ is adjacent to $c, v, y$ and non-adjacent to $s_{2}, i_{12}$. Thus $v$ must be adjacent to $c$ because otherwise $v i_{12} s_{2} c z$ would induce a $C_{5}$. Furthermore, $y$ is non-adjacent to $s_{2}$ (otherwise $v y z s_{2} i_{2} i_{12}$ would induce a $\mathfrak{A}_{11}$ (antenna) and hence non-adjacent to $c$ (otherwise $v y z s_{2} i_{12} c$ would induce a $\mathfrak{A}_{23}$. But now either $v c z s_{2} i_{2} i_{12}$ induces a $\mathfrak{A}_{11}$ (antenna) (if $i_{2} c \notin E$ ) or $v c z s_{2} i_{2} i_{12} y$ induces a $\mathfrak{A}_{17}\left(\right.$ if $i_{2} c \in E$ ).

2. $I_{2}(b) S_{12}$

Consider an edge $i_{2} s_{12}$ with $i_{2} \in I_{2}, s_{12} \in S_{12}$. We may assume that $x i_{2} \notin E$, because otherwise the previous case applies. $v c \in E$ because otherwise $v i_{12} s_{2} c s_{12}$ would induce a $C_{5}$ and hence $i_{2} c \in E$ because otherwise $v i_{12} s_{2} c s_{12} i_{2}$ would induce a $\mathfrak{A}_{11}$ (antenna). Now, if $y c \notin E$ then either vxycs $i_{2} i_{2} s_{12} i_{12}$ induces a $\mathfrak{A}_{19}$ (if $y s_{2} \notin E$ ) or vxycs $_{2} i_{2} s_{12}$ induces a $\mathfrak{A}_{27}$ If $y c \in E$ then either vxycs $s_{2} i_{12}$ induces a $\mathfrak{A}_{23}\left(\right.$ if $y s_{2} \notin E$ ) or $v x y c s_{2} i_{2} s_{12} i_{12}$ induces a $\mathfrak{A}_{40}\left(\right.$ if $y s_{2} \in E$ ).

Lemma 23 If $S_{2}$ (Ø) $I_{12}$ and $I_{2}$ (Ø) $\left(S_{11}+x\right)$ then $G$ is probe split. 
Proof: We show that $(Q, U, J)$ with

$$
\begin{aligned}
& Q=C+v, \\
& U=S_{1} \cup\{x\} \cup\left(I_{12} \cap N\left(S_{2}\right)\right), \\
& J=S_{2} \cup I \backslash\left(I_{12} \cap N\left(S_{2}\right)\right)
\end{aligned}
$$

is a valid probe split partition. By assumption $S_{2} \neq \emptyset$, therefore $v$ (1) $C$ and $Q$ is a clique. By Lem. 18, $U$ is independent and by Lem. 22, $U$ (1) $Q$. Finally, by Lem.21.a. $J$ is independent.

Lemma 24 If $I_{2}\left(\emptyset S_{12}\right.$ and $S_{2} \emptyset\left(I_{11}+y\right)$ then $\mathrm{G}$ is probe split.

Proof: We show that $(Q, U, J)$ with

$$
\begin{aligned}
& Q=C+v, \\
& U=\left(I_{11}+y\right) \cup\left(I_{12} \cap N\left(S_{2}\right)\right) \cup S_{12}, \\
& J=\left(S \backslash S_{12}\right) \cup\left(I_{12} \backslash N\left(S_{2}\right)\right) \cup I_{2}
\end{aligned}
$$

is a valid probe split partition. Again is by assumption $S_{2} \neq \emptyset$ and thus $Q$ a clique. By Lem. 18, $U$ is independent and by Lem. 21.b $J$ is independent. First we show that

$$
\left(\left(I_{11}+y\right) \cap N\left(S_{2}\right)\right) \text { (1) } C .
$$

Let $z \in\left(I_{11}+y\right) \cap N\left(S_{2}\right)$ be non-adjacent to $c \in C$ and adjacent to $s_{2} \in S_{2}$. Let $i_{2} \in I_{2}$ and $s_{12} \in S_{12}$ be neighbours, and note that by Lem. 21.b, $i_{2} x \notin E$. But now either $v c z s_{2} s_{12} i_{2}$ induce a $\mathfrak{A}_{11}$ (antenna), or $v c z s_{2} s_{12} i_{2} x$ induce a $\mathfrak{A}_{27}$. This proves $[17)$.

Next we show that

$$
\left(\left(I_{11}+y\right) \backslash N\left(S_{2}\right)\right) \text { (1) } C \text {. }
$$

Let $z \in\left(I_{11}+y\right) \backslash N\left(S_{2}\right)$ be non-adjacent to $c \in C$. Let $i_{2} \in I_{2}$ and $s_{12} \in S_{12}$ be neighbours, and let $s_{2} \in S_{2}$ and $t \in I_{11}+y$ be neighbours as well. Note that $t \neq z$ and that by (17) $t c \in E$. But now vcxzts $s_{12} i_{2}$ induce either a $\mathfrak{A}_{29}\left(\right.$ if $c i_{2} \notin E$ ) or a $\mathfrak{A}_{30}$ (if $c i_{2} \in E$ ).

Lemma 25 If $I_{2}$ (b) $S_{12}$ and $S_{2}$ (1) $\left(I_{11}+y\right)$ then $G$ is probe split.

Proof: We distinguish two cases:

1. $v$ (1) $C$

We show that $(Q, U, J)$ with

$$
\begin{aligned}
Q & =C+v, \\
U & =S_{1} \cup\left(I_{12} \cap N\left(S_{2}\right)\right), \\
J & =\left(I \backslash N\left(S_{2}\right)\right) \cup S_{2}
\end{aligned}
$$

is a valid probe split partition. By assumption $v$ (1) $C$ and thus $Q$ is a clique. By Lem. $18, U$ is independent and by Lem. 22, $U$ (1) $Q$. Finally, by assumption $J$ is independent. 
2. $v(1) C$

By the discussion after the definition of $S_{2}$, we have that $S_{2}=\emptyset$ and that $v$ is non-adjacent to precisely one vertex $c \in C$. Furthermore, $c$ (0) $I_{2}$, because $\bar{N}(v)$ is assumed to be independent. We start by showing that

$$
\text { if } c(\emptyset)\left(I_{11}+y\right) \text { then } x \text { (1) }\left(I_{2} \cap N\left(S_{12}\right)\right) \wedge(C-c) \text { (1) }\left(I_{2} \cap N\left(S_{12}\right)\right) \wedge S_{11}=\emptyset \text {. }
$$

Let $z \in N(c) \cap\left(I_{11}+y\right)$, and $i_{2} \in I_{2}$ with a neighbour $s_{12} \in S_{12}$. Then $x i_{2} \in E$, and thus $x$ (1) $\left(I_{2} \cap N\left(S_{12}\right)\right)$, because otherwise $c v x z i_{2} s_{12}$ induces a $\mathfrak{A}_{9}$ (co-fish) Next, assume $c^{\prime} \in C-c$ is non-adjacent to $i_{2}$. But then $c v x z i_{2} s_{12} c^{\prime}$ induce either a $\mathfrak{A}_{10}$ (if $z c^{\prime} \notin E$ ) or a $\mathfrak{A}_{18}\left(\right.$ if $z c^{\prime} \in E$ ). Thus $(C-c)$ (1) $\left(I_{2} \cap N\left(S_{12}\right)\right)$. Finally, assume a vertex $s_{11} \in S_{11}$ exists. If $y c \notin E$ then $y \neq z$ and $x y v c z s_{11}$ induce a $\mathfrak{A}_{26}\left(\overline{P_{6}}\right)$ If $y c \in E$ then either $x y v c s_{11} i_{2} s_{12}$ induce a $\mathfrak{A}_{21}$ (if $s_{11} i_{2} \in E$ ) or yvcs $_{11} i_{2} s_{12}$ induce a $\mathfrak{A}_{9}$ (co-fish) (if $s_{11} i_{2} \notin E$ ). Thus $S_{11}=\emptyset$, finishing the proof of (19).

Next we show that

$$
\text { if }(C-c)(1)\left(I_{12} \cap N(c)\right) \text { then } S_{11}=\emptyset \wedge x(1)\left(I_{2} \cap N\left(S_{12}\right)\right) \wedge(C-c)(1)\left(I_{2} \cap N\left(S_{12}\right)\right) \text {. }
$$

By (19), we may assume that $c$ (0) $I_{11}+y$. Let $i_{12} \in\left(I_{12} \cap N(c)\right)$ be non-adjacent to $c^{\prime} \in C-c$. First, assume a vertex $s_{11} \in S_{11}$ exists. Then $c c^{\prime} v x y i_{12} s_{11}$ induce either a $\mathfrak{A}_{18}$ (if $y c^{\prime} \in E$ ) or a $\mathfrak{A}_{10}$ (if $y c^{\prime} \notin E$ ). Thus $S_{11}=\emptyset$. Consider now neighbours $i_{2} \in I_{2}$ and $s_{12} \in S_{12}$. If $x i_{2} \notin E$ then $v c c^{\prime} x y i_{12} i_{2} s_{12}$ induce a $\mathfrak{A}_{38}\left(\right.$ if $c^{\prime} i_{2}, c^{\prime} y \notin E$ ), a $\mathfrak{A}_{42}\left(\right.$ if $c^{\prime} i_{2} \in E, c^{\prime} y \notin E$ ) a $\mathfrak{A}_{41}$ (if $c^{\prime} i_{2} \notin E, c^{\prime} y \in E$ ) or a $\mathfrak{A}_{45}$ (if $c^{\prime} i_{2}, c^{\prime} y \in E$ ). Thus $x$ (1) $\left(I_{2} \cap N\left(S_{12}\right)\right.$ ). It follows that $c^{\prime}$ (1) $I_{2} \cap N\left(S_{12}\right)$ because otherwise $c c^{\prime} v x s_{12} i_{12} i_{2}$ induce a $\mathfrak{A}_{20}$. Finally, assume that a vertex $c^{\prime \prime} \in C \backslash\left\{c, c^{\prime}\right\}$ is non-adjacent to a vertex $i_{2}^{\prime} \in I_{2} \cap N\left(S_{12}\right)$. Let $s_{12}^{\prime} \in S_{12}$ be a neighbour of $i_{2}^{\prime}$ (note that $i_{2}^{\prime}=i_{2}$ or $s_{12}^{\prime}=s_{12}$ is possible). $c^{\prime} i_{2}^{\prime} \in E$ because $c^{\prime}$ (1) $I_{2} \cap N\left(S_{12}\right)$ and $c^{\prime \prime} i_{12} \in E$ because otherwise $c c^{\prime \prime} v x s_{12}^{\prime} i_{12} i_{2}^{\prime}$ would induce a $\mathfrak{A}_{20}$. But then $c c^{\prime} c^{\prime \prime} v x s_{12}^{\prime} i_{12} i_{2}^{\prime}$ induce a $\mathfrak{A}_{46}$. This finishes the proof of 20).

We are now ready to prove the lemma. If $c(6)\left(I_{11}+y\right)$ or $(C-c)(1)\left(I_{12} \cap N(c)\right)$ then by [19], 20], $(Q, U, J)$ with

$$
\begin{aligned}
Q & =C-c+x, \\
U & =\{c, v\} \cup\left(I_{2} \cap N\left(S_{12}\right)\right), \\
J & =I \backslash\left(I_{2} \cap N\left(S_{12}\right)\right) \cup S_{12}
\end{aligned}
$$

is a valid probe split partition. Otherwise $(Q, U, J)$ with

$$
\begin{aligned}
Q & =C-c+v, \\
U & =S \cup\left(I_{12} \cap N(c)\right), \\
J & =I \backslash\left(I_{12} \cap N(c)\right) \cup\{c\}
\end{aligned}
$$

is a valid probe split partition.

Lemma 26 If $I_{2}$ (0) $S_{12}$ and $S_{2}$ (b) $\left(I_{11}+y\right)$ then $G$ is probe split. 
Proof: Let

$$
\begin{aligned}
& A=\left(I_{11}+y\right) \cap N\left(S_{2}\right), \\
& B=\left(I_{11}+y\right) \backslash A .
\end{aligned}
$$

By assumption, $A \neq \emptyset$ and $S_{2} \neq \emptyset$ and thus $v$ (1) $C$. We first show that

$$
\text { Either } A \text { (1) } C \text { or } \exists ! c_{A} \in C: c_{A} \text { (1) } A \wedge\left(C-c_{A}\right) \text { (1) } A \text {. }
$$

Let $z \in\left(I_{11}+y\right) \cap N\left(S_{2}\right)$ be non-adjacent to $c_{1}, c_{2} \in C\left(c_{1} \neq c_{2}\right)$, then $z x v c_{1} c_{2}$ and a neighbour of $z$ in $S_{2}$ induce a $\overline{\mathfrak{A}_{24}\left(\overline{2 P_{3}}\right)}$ This proves that every vertex in $\left(I_{11}+y\right) \cap N\left(S_{2}\right)$ has at most one non-neighbour in $C$. Consider two vertices $z, z^{\prime} \in\left(I_{11}+y\right) \cap N\left(S_{2}\right)$ and $c \in C$ such that $z c \in E$ but $z^{\prime} c \notin E$. If $z$ and $z^{\prime}$ have a common neighbour $s \in S_{2}$ then $v z z^{\prime} c x s$ induce a $\mathfrak{A}_{24}\left(\overline{2 P_{3}}\right)$ and if they have private neighbours $s, s^{\prime}$, respectively, then $v z z^{\prime} c x s s^{\prime}$ induce a $\mathfrak{A}_{25}$. This proves that every two vertices in $A$ have the same neighbours in $C$, thereby implying the validity of (21).

We now distinguish two cases:

1. $S_{2}$ (1) $I_{12}$

If $S_{11}=\emptyset$ then $(Q, U, J)$ with

$$
\begin{aligned}
Q & =(C \cap N(A)) \cup\{v, x\}, \\
U & =A \cup(C \backslash N(A)), \\
J & =(I \backslash A) \cup(S-x)
\end{aligned}
$$

is a valid probe split partition: It is clear that $Q$ is a clique. By 21) $U$ is an independent set and $Q$ (1) $U$. Since it is assumed that $I_{12}$ (1) $S_{2}$ and $S_{2}$ (1) $I_{12}, J$ is an independent set.

So, assume that $S_{11} \neq \emptyset$. Since $v$ (1) $C$, by Lem. 19, y (1) $C$. If $A=\{y\}$, then $S_{2}$ (1) $I_{11}$ and thus $(Q, U, J)$ with

$$
\begin{aligned}
Q & =C \cup\{v, y\} \\
U & =S_{11}+x, \\
J & =(I-y) \cup S_{12} \cup S_{2}
\end{aligned}
$$

is a valid probe split partition.

So, assume that $A-y \neq \emptyset$, then, by Lem. 20, $I_{2}$ (1) $S_{11}$. Note that $A$ (1) $C$ both when $y \in A$ and when $y \notin A$ : If $y \in A$ then this follows from $y$ (1) $C$ and (21); if $y \notin A$ and $a \in A$ is non-adjacent to $c \in C$ and adjacent to $s \in S_{2}$ then avcys together with a vertex from $S_{11}$ induce a $\mathfrak{A}_{23}$. Now we can show that $(Q, U, J)$ with

$$
\begin{aligned}
Q & =C \cup\{v, x\}, \\
U & =A+y, \\
J & =(S-x) \cup(I \backslash(A+y))
\end{aligned}
$$

is a valid probe split partition (note that $y$ may or may not be in $A$ ): We have demonstrated that $A$ (1) $C$ and $y$ (1) $C$ and thus $Q$ (1) $U$. By assumption $I_{2}$ (1) $S_{12}$ and $S_{2}$ (0) $I_{12}$, we have demonstrated that $I_{2}$ (1) $S_{11}$, and thus $J$ is an independent set. 
2. $S_{2}\left(\right.$ (b) $I_{12}$

By Lem. 23 we may assume that $I_{2}$ (0) $\left(S_{11}+x\right)$. We first show

$$
\forall i_{12} \in I_{12} \cap N\left(S_{2}\right) \forall z \in I_{11}+y: N\left(i_{12}\right) \cap C \subseteq N(z) \cap C .
$$

Let $i_{12} \in I_{12}, z \in I_{11}+y, c \in C$ and assume $i_{12} c \in E$ but $z c \notin E$. Let $s_{2} \in S_{2}$ be a neighbour of $i_{12}$. First consider the case $z \in A$ and let $s_{2}^{\prime} \in S_{2}$ be a neighbour of $z$. Then either $v c x z i_{12}$ and

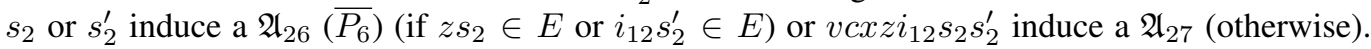
Next consider the case $z \notin A$. Let $a \in A$ with a neighbour $s_{2}^{\prime} \in S_{2}$ (by assumption $A \neq \emptyset$ ), then by the previous case $a c \in E$. But then either $v c x a z i_{12}$ and $s_{2}$ or $s_{2}^{\prime}$ induce a $\mathfrak{A}_{28}$ (if $a s_{2} \in E$ or $i_{12} s_{2}^{\prime} \in E$ ), or $v c x a z i_{12} s_{2} s_{2}^{\prime}$ induce a $\mathfrak{A}_{30}$ (otherwise). Thus (22) holds.

Next we show

$$
\begin{gathered}
B \text { (1) } C \backslash N(A), \\
B \text { (1) } C \cap N(A), \\
I_{11 \neq \emptyset \longrightarrow}\left(I_{12} \cap N\left(S_{2}\right)\right) \text { (1) }(C \cap N(A)) .
\end{gathered}
$$

Suppose $z \in B$ is adjacent to $c_{A}$ (remember that $C \backslash N(A)=\left\{c_{A}\right\}$ ). Let $a \in A$ and let $s_{2} \in S$, $i_{12} \in I_{12}$ be neighbours. By [22], $c_{A} i_{12} \notin E$ and thus $v c x z i_{12} s_{2}$ induce a $\mathfrak{A}_{23}$ This proves the first statement.

Let $a \in A, b \in B, c \in C-c_{A}$ and assume $c b \notin E$. Let $s_{2}^{\prime} \in S_{2}$ and $i_{12} \in I_{12}$ be neighbours. If $i_{12} c \notin E$ then either $v x c a i_{12} s_{2}^{\prime}$ induce a $\mathfrak{A}_{23}\left(\right.$ if $a s_{2}^{\prime} \notin E$ ) or $v x c a b i_{12} s_{2}^{\prime}$ induce a $\mathfrak{A}_{25}$ (if $a s_{2}^{\prime} \in E$ ). If $i_{12} c \in E$ then $v x c a b i_{12} s_{2}^{\prime}$ induce a $\mathfrak{A}_{28}$ (if $a s_{2}^{\prime} \in E$ ) or $v x c a b i_{12} s_{2}^{\prime} s_{2}$ induce a| $\mathfrak{A}_{30}$ (if $a s_{2}^{\prime} \notin E$ and with $s_{2} \in S_{2}$ a neighbour of $a$ ). This proves the second statement.

Finally assume $i_{12} \in I_{12} \cap N\left(S_{2}\right)$ with neighbour $s_{2} \in S_{2}$ is non-adjacent to $c \in C \cap N(A)$. We will show that $I_{11}=\emptyset$. Let $i_{11} \in I_{11}$, then, by the second statement, $y c \in E$ and $i_{11} c \in E$. If $y s_{2} \notin E$ or $i_{11} \notin E$ then $v x c s_{2} i_{12} y$ or $v x c s_{2} i_{12} i_{11}$, respectively, induce a $\mathfrak{A}_{23}$, otherwise $v x c s_{2} i_{12} y i_{11}$ induce a $\mathfrak{A}_{18}$. This finishes the proof of 23).

Note that (23) implies that all vertices in $I_{11}+y$ are adjacent to $C-c_{A}$ and non-adjacent to $c_{A}$. The last intermediate result we need is

$$
\text { either }\left(I_{12} \cap N\left(S_{2}\right)\right) \text { (1) }(C \cap N(y)) \text { or }\left(S_{2} \cap N\left(I_{12}\right)\right) \text { (1) } y \wedge C \text { (1) } y \text {. }
$$

Assume $i_{12} \in I_{12} \cap N\left(S_{2}\right)$ is non-adjacent to $c \in C \cap N(y)$, and let $s_{2} \in S_{2}$ be adjacent to $i_{12}$. First we show that $S_{2} \cap N\left(I_{12}\right)$ (1) $y$. Assume $s_{2}^{\prime} \in S_{2} \cap N\left(I_{12}\right)$ is non-adjacent to $y$ and let $i_{12}^{\prime} \in I_{12}$ be a neighbour of $s_{2}^{\prime}$. Now $y s_{2} \in E, i_{12}^{\prime} c \in E$ and $i_{12} s_{2}^{\prime} \notin E$, because otherwise $v c x y$ and, respectively, $i_{12} s_{2}, i_{12}^{\prime} s_{2}^{\prime}, i_{12} s_{2}^{\prime}$ would induce a $\mathfrak{A}_{23}$. But this implies $s_{2} \neq s_{2}^{\prime}, i_{12} \neq i_{12}^{\prime}$ and thus vcxys $s_{2} s_{2}^{\prime} i_{12} i_{12}^{\prime}$ either induce a $\mathfrak{A}_{40}\left(\right.$ if $i_{12}^{\prime} s_{2} \notin E$ ) or a $\mathfrak{A}_{45}\left(\right.$ if $i_{12}^{\prime} s_{2} \in E$ ). Next we show that $C$ (1) $y$. Assume not, then $y c^{\prime} \notin E$ for some $c^{\prime} \in C\left(c^{\prime} \neq c\right)$. If $i_{12} c^{\prime} \notin E$ then $v x y c c^{\prime} i_{12} s_{2}$ induce

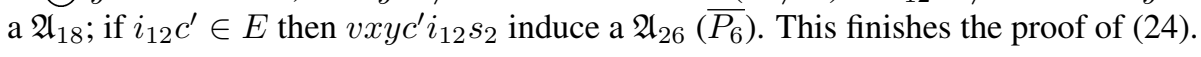

For the remainder of the proof of the lemma we consider two cases:

(a) $I_{12} \cap N\left(S_{2}\right)$ (1) $C-c_{A}$ 
We show that $(Q, U, J)$ with

$$
\begin{aligned}
Q & =C-c_{A}+v, \\
U & =\left(I_{11}+y\right) \cup\left(I_{12} \cap N\left(S_{2}\right)\right) \cup\left\{c_{A}\right\}, \\
J & =S \cup\left(I_{12} \backslash N\left(S_{2}\right)\right) \cup I_{2}
\end{aligned}
$$

is a valid probe split partition (note that $c_{A}$ might not exist). It is clear that $Q$ is a clique and that $J$ is independent. By (21), 22) and 23), $U$ is independent and by (23) and the case distinction $U$ (1) $Q$.

(b) $I_{12} \cap N\left(S_{2}\right)(1)\left(C-c_{A}\right)$

By [23, $I_{11}=\emptyset$, and by (24), $\left(S_{2} \cap N\left(I_{12}\right)\right)$ (1) $y$ and $C$ (1) $y$. Thus $(Q, U, J)$ with

$$
\begin{aligned}
Q & =C+y, \\
U & =\{v\} \cup\left(S_{2} \cap N\left(I_{12}\right)\right), \\
J & =I_{2} \cup\left(S \backslash\left(S_{2} \cap N\left(I_{12}\right)\right)\right)
\end{aligned}
$$

is a valid probe split partition.

Lemma 27 If $I_{2}$ (9) $S_{12}$ and $S_{2}$ (9) $\left(I_{11}+y\right)$, then $G$ is probe split.

\section{Proof:}

1. $I_{12}$ (0) $S_{2}$

We may assume $v$ (1) $C$ because otherwise $\left(C+v, S \backslash S_{2}, I \cup S_{2}\right)$ is a valid probe split partition. So let $c \in C$ be the single non-neighbour of $v$ in $C$. Then $S_{2}=\emptyset$ and $c$ (0) $I_{2}$. We may also assume that $S_{11} \neq \emptyset$ because otherwise $\left(C-c+x,\{c, v\}, I \cup S_{12}\right)$ is a valid probe split partition. We start by proving

$$
\text { if } y c \notin E \text { then } c\left(I_{11} \wedge(C-c) \text { (1) }\left(I_{12} \cap N(c)\right)\right. \text {. }
$$

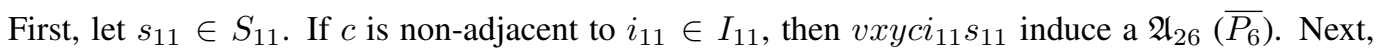
if there are neighbours $s_{11} \in S_{11}, i_{12} \in I_{12}$ such that $c^{\prime} i_{12} \notin E$, for some $c^{\prime} \in C-c$, then vxycc' $i_{12} s_{11}$ induce a $\mathfrak{2}_{18}$. This proves 25.

By [25), $(Q, U, J)$ with

$$
\begin{aligned}
Q & =C-c+v, \\
U & =S \cup\left(I_{12} \cap N(c)\right), \\
J & =I \backslash\left(I_{12} \cap N(c)\right)+c
\end{aligned}
$$

is a valid probe split partition if $y c \notin E$. So assume $y c \in E$. We may then also assume $I_{2}$ (b) $S_{11}$ because otherwise $(C-c+x+y,\{c, v\}, I \cup S)$ is a valid probe split partition. We prove that

$$
\text { if } I_{11} \neq \emptyset \text { then } c \text { (0) }\left(I_{11} \cup I_{12}\right) \wedge S_{12}=\emptyset \text {. }
$$


Let $i_{2} \in I_{2}$ and $s_{11} \in S_{11}$ be neighbours. First, consider a vertex $i_{11} \in I_{11}$ that is adjacent to $c$. Then either $v x c s_{11} i_{2} i_{11}$ induce a $\mathfrak{A}_{9}$ (co-fish) (if $x i_{2} \notin E$ ) or $v x y c s_{11} i_{2} i_{11}$ induce a $\mathfrak{A}_{10}$ (otherwise). Next, assume $i_{12} \in I_{12}$ is adjacent to $c$. Let $i_{11} \in I_{11}$, then $c i_{11} \notin E$. Now either vxyci $i_{11} s_{11} i_{2} i_{12}$ induce a $\mathfrak{A}_{38}$ (if $x i_{2} \notin E$ ) or vxycs $_{11} i_{2} s_{12}$ induce a $\mathfrak{A}_{20}$ (if $x i_{2} \in E$ ). Finally assume $s_{12} \in S_{12}$ exists. Let $i_{11} \in I_{11}$, then $c i_{11} \notin E$. Now either vxyci $i_{11} s_{11} i_{2} s_{12}$ induce a $\mathfrak{A}_{38}$ (if $x i_{2} \notin E$ ) or $v x y c s_{11} i_{2} s_{12}$ induce a $\mathfrak{A}_{20}$ (if $x i_{2} \in E$ ). This finishes the proof of 26 .

By (26), $(C-c+v+y, S, I-y+c)$ is a valid probe split partition if $I_{11} \neq \emptyset$. Now we assume $I_{11}=\emptyset$.

$$
\text { If } S_{12} \neq \emptyset \text { then } x \text { (0) } I_{2} \text {. }
$$

Let $s_{12} \in S_{12}$. First, if $i_{2} \in I_{2} \cap N\left(S_{11}\right)$ with neighbour $s_{11} \in S_{11}$ is adjacent to $c$, then vxycs $_{12} i_{2} s_{11}$ induce either a $\mathfrak{A}_{20}$ (if $c i_{2} \notin E$ ) or a $\mathfrak{A}_{10}$ (if $c i_{2} \in E$ ). Next, let $i_{2} \in I_{2}$ be adjacent to $s_{11} \in S_{11}$ and assume $i_{2}^{\prime} \in I_{2}$ is non-adjacent to $x$. But then vxycs $_{12} i_{2} s_{11} i_{2}^{\prime}$ induce either a $\mathfrak{A}_{36}$ (if $c i_{2}, c i_{2}^{\prime} \notin E$ ) or a $\mathfrak{A}_{39}$ (if $c i_{2}, c i_{2}^{\prime} \in E$ ) or a $\mathfrak{A}_{38}$ (otherwise). This finishes the proof of 27).

$$
\text { If } S_{12} \cup\left(I_{12} \cap N(c)\right) \neq \emptyset \text { then } \exists ! s_{i} \in S_{11}: S_{11} \cap N\left(I_{2}\right)=\left\{s_{i}\right\} \text {. }
$$

We already know we may assume $\left|S_{11} \cap N\left(I_{2}\right)\right| \geq 1$. Let $t \in S_{12} \cup\left(I_{12} \cap N(c)\right)$ and $s, s^{\prime} \in$ $S_{11} \cap N\left(I_{2}\right)$. If $s, s^{\prime}$ have a common neighbour $i_{2} \in I_{2}$ then vyctss ${ }^{\prime} i_{2}$ induce a $\mathfrak{A}_{20}$, if they have private neighbours $i_{2}, i_{2}^{\prime} \in I_{2}$, respectively, then $v y c t s s^{\prime} i_{2} i_{2}^{\prime}$ induce a $\mathfrak{A}_{36}$ This finishes the proof of 28).

We are now ready to prove the first case of the lemma, distinguishing two cases:

(a) $S_{12} \neq \emptyset$

We claim that $(Q, U, J)$ with

$$
\begin{aligned}
Q & =C-c+y+s_{i}, \\
U & =\{v, c\}, \\
J & =(I-y) \cup\left(S_{11}-s_{i}+x\right) \cup S_{12}
\end{aligned}
$$

is a valid probe split partition. By Lem. 19, $Q$ is a clique and by assumption $U$ is independent. By (27), $x$ is non-adjacent to $J-x$ and by [28), $S_{11}-s_{i}$ is non-adjacent to $I$, thus $J$ is independent.

(b) $S_{12}=\emptyset$

If $I_{12} \cap N(c)=\emptyset$, then is $\left(C-c+v+y, S_{11}-x, I-y+c\right)$ a valid probe split partition. Otherwise $(Q, U, J)$ with

$$
\begin{aligned}
Q & =C-c+y+s_{i}, \\
U & =\{v, c\}, \\
J & =(I-y) \cup\left(S_{11}-s_{i}+x\right)
\end{aligned}
$$

is a valid probe split partition. 
2. $I_{12}$ (b) $S_{2}$

Since $S_{2} \neq \emptyset, v$ (1) $C$. We may assume $I_{2}$ (0) $\left(S_{11}+x\right)$, because otherwise by Lem. 22. $(Q, U, J)$ with

$$
\begin{aligned}
Q & =C+v, \\
U & =\left(S \backslash S_{2}\right) \cup\left(I_{12} \cap N\left(S_{2}\right)\right), \\
J & =I \backslash\left(I_{12} \cap N\left(S_{2}\right)\right) \cup S_{2}
\end{aligned}
$$

is a valid probe split partition. Furthermore, if $S_{11} \neq \emptyset$, then by Lem. 19 , y (1) $C$. Then $C$ (1) $\left(I_{12} \cap\right.$ $N\left(S_{2}\right)$ ), because otherwise $v x y c i_{12} s_{2}$, with neighbours $i_{12} \in I_{12}$ and $s_{2} \in S_{2}$ such that $c i_{12} \notin E$, would induce a $\mathfrak{2}_{23}$, and again $(Q, U, J)$ from 29 is a valid probe split partition. Thus, we may also assume $S_{11}=\emptyset$ and $C(1)\left(I_{12} \cap N\left(S_{2}\right)\right)$.

First, observe that

$$
\forall c \in C: c(1)\left(I_{12} \cap N\left(S_{2}\right)\right) \rightarrow c y \notin E:
$$

If $i_{12} \in I_{12} \cap N\left(S_{2}\right)$ with neighbour $s_{2} \in S_{2}$ and non-neighbour $c \in C$, then $v x y z i_{12} s_{2}$ induce a $\mathfrak{A}_{23}$. We now prove

$$
\exists ! c \in C:(C-c)(1)\left(I_{12} \cap N\left(S_{2}\right)\right) \wedge c(0)\left(I_{11}+y\right) \wedge c(0)\left(I_{12} \cap N\left(S_{2}\right)\right) \wedge C-c(1)\left(I_{11}+y\right)
$$

Let $i_{12} \in I_{12} \cap N\left(S_{2}\right)$ with neighbour $s_{2} \in S_{2}$ and non-neighbour $c \in C$. By (30), yc $\notin E$. If $c^{\prime} \in C-c$ exists such that $c^{\prime}(1)\left(I_{12} \cap N\left(S_{2}\right)\right)$, then $c^{\prime} y \notin E$ and $v x y c c^{\prime} i_{12} s_{2}$ either induce a $\mathfrak{A}_{25}$ (if $c^{\prime} i_{12} \notin E$ ), or a $\mathfrak{A}_{28}$ (if $c^{\prime} i_{12} \in E$ ). Thus, $(C-c)$ (1) $\left(I_{12} \cap N\left(S_{2}\right)\right)$. Next, assume $c$ is adjacent to $i_{11} \in I_{11}$. Let $i_{12} \in I_{12}$ be non-adjacent to $c$ with neighbour $s_{2} \in S_{2}$. But then $v x c i_{11} i_{12} s_{2}$ induce a $\mathfrak{A}_{23}$ So $c$ (0) $I_{11}$, and by (30), $c$ (0) $y$. Now assume $i_{12}^{\prime} \in I_{12} \cap N\left(S_{2}\right)$ exists such that $c i_{12}^{\prime} \in E$. Let $s_{2}^{\prime} \in S_{2}$ be adjacent to $i_{12}^{\prime}$ (possibly $s_{2}=s_{2}^{\prime}$ ). If $s_{2}=s_{2}^{\prime}$ then $x y v c i_{12} i_{12}^{\prime} s_{2}$ induce a $\mathfrak{A}_{27}$, otherwise $x y v c i_{12} i_{12}^{\prime} s_{2} s_{2}^{\prime}$ induce a $\mathfrak{A}_{19}$. Thus $c$ (0) $I_{12} \cap N\left(S_{2}\right)$. Finally, assume $z \in I_{11}+y$ is non-adjacent to $c^{\prime} \in C-c$. Then $v x z c c^{\prime} i_{12} s_{2}$ induce a $\mathfrak{A}_{28}$. Thus $(C-c)(1)\left(I_{11}+y\right)$, finishing the proof of 31.

We claim that $(Q, U, J)$ with

$$
\begin{aligned}
Q & =C-c+v, \\
U & =\left(I_{11}+y+c\right) \cup\left(I_{12} \cap N\left(S_{2}\right)\right), \\
J & =S \cup I_{2} \cup\left(I_{12} \backslash N\left(S_{2}\right)\right)
\end{aligned}
$$

is a valid probe split partition. It is clear that $Q$ is a clique. By (31), $U$ is independent and $U$ (1) $Q$ and by assumption $J$ is independent.

Lemma 28 (Prop. 8) If $G-v$ is a probe split graph, then so is $G$. Moreover, a probe split partition of $G$ can be obtained from a probe split partition of $G-v$ in linear time.

Proof: Lem. 24 to 27 cover all possible relations between $I_{2}$ and $S_{12}$ and between $S_{2}$ and $I_{11}+y$ and therefore prove that if $G-v$ is probe split, then so is $G$. The proofs of those lemmas also show how a probe split partition of $G$ can be calculated from a probe split partition of $G-v$. It is straightforward that this can be done in linear time using suitable data structures. 


\section{Partitioned probe split graphs}

In this section we investigate graphs $G=(N, P, E)$ with a given partition of its vertex set into probes $P$ and non-probes $N$, with $G[N]$ an independent set.

Definition 29 A graph $G=(N, P, E)$, with $N$ an independet set, is a partitioned probe split graph (with respect to the partition $V(G)=N \cup P$ ) if there exist $E^{\prime} \subseteq N \times N$ such that $G^{\prime}=\left(N \cup P, E \cup E^{\prime}\right)$ is a split graph.

First we prove a characterisation by 10 forbidden induced subgraphs for partitioned probe split graphs, and then we give a polynomial recognition algorithm.

In this section we shall write $X_{N}, X_{P}$ for the intersection of a vertex set $X$ with $N, P$, respectively in a partitioned probe split graph $(N, P, E)$.

Lemma 30 If $G=(N, P, E)$ is a partitioned probe split graph and $(C, S, I)$ is a valid probe split partition for $G$, then for all $x \in I$ either $|S \cap N(x)| \leq 1$, or $x$ (1) $C_{P}$, or $x \notin N \wedge S_{P} \cap N(x)=\emptyset$.

Proof: Let $x \in I$ be adjacent to $s_{1}, s_{2} \in S$ and non-adjacent to $c \in C_{P}$. Then $x \notin N$ because otherwise $x c s_{1} s_{2}$ would induce a $\mathfrak{B}_{4}$ and $s_{1}$ and $s_{2}$ are both in $N$, because otherwise $x c s_{1} s_{2}$ would induce a $\mathfrak{B}_{3}$ or $\mathrm{a} \mathfrak{B}_{4}$
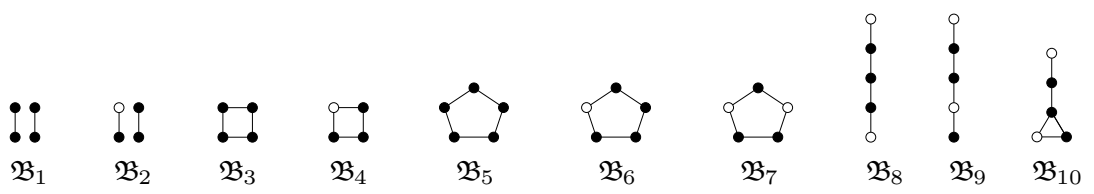

Fig. 2: Forbidden subgraphs for partitioned probe split graphs (black vertices probes, white vertices nonprobes).

Theorem $31 G=(N, P, E)$ with $N$ an independent set is a partitioned probe split graph iff $G$ has none of the graphs of Fig. 2 as an induced subgraph.

Proof: It is clear that if $G$ is probe split, then $G$ does not contain any of the graphs of Fig. 2 as an induced subgraph. For the other direction it is easy to verify that if $G$ does not contain any of the graphs in Fig.2. then $G$ does not contain any of the graphs in Fig. 1 either, and thus $G$ is probe split. Let $(C, S, I)$ be a probe split partition of $G$. We distinguish two cases.

1. $S_{P}$ (0) $I_{P}$

Let

$$
\begin{aligned}
& A=\left\{v \in I_{N} \cap N\left(S_{P}\right) \mid v(1) C_{P}\right\}, \\
& B=I_{N} \cap N\left(S_{P}\right) \backslash A .
\end{aligned}
$$


Suppose $B=\emptyset$. If we add all edges between vertices in $N^{\prime}=S_{N} \cup A \cup C_{N}$ then we get a split graph with clique $Q=N^{\prime} \cup C_{P}$ and independent set $V(G) \backslash Q$. As $N^{\prime} \subseteq N$, it follows that $G$ is probe split with respect to the partition $(N, P)$.

So assume $B \neq \emptyset$. Then

$$
S_{N}(1) I_{P}:
$$

Let $n \in S_{N}$ be adjacent to $i \in I_{P}$ and let $b \in B$ with neighbour $s \in S_{P}$ and non-neighbour $c \in C_{P}$.

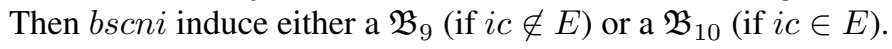

Moreover

$$
\left|S_{P} \cap N(B)\right| \leq 1
$$

To see this, note first that by Lem. 30 and definition of $B$, every vertex in $B$ has exactly one neighbour in $S_{P}$. Suppose now that vertices $s, s^{\prime} \in S_{P} \cap N(B)$ exist $\left(s \neq s^{\prime}\right)$, with neighbour $b, b^{\prime} \in B\left(b \neq b^{\prime}\right)$, respectively. Let $c \in C_{P}$ be non-adjacent to $b$. Then $b^{\prime} s^{\prime} c s b$ induce either a $\mathfrak{B}_{8}$ (if $b^{\prime} c \notin E$ ) or a $\mathfrak{B}_{10}$ (if $b^{\prime} c \in E$ ). Contradiction.

Let $\left\{s_{B}\right\}=S_{P} \cap N(B)$. Then

$$
s_{B} \text { (1) } A \text { : }
$$

Suppose $s_{B}$ is non-adjacent to $a \in A$. Let $b \in B$ be non-adjacent to $c \in C_{P}$ and let $s \in S_{P}$ be a neighbour of $a$. Then $b s_{B} c s a$ induce a

Now, if we add all edges between vertices in $N^{\prime}=A \cup C_{N}$ then we get a split graph with clique $Q=N^{\prime} \cup C_{P}+s_{B}$ and independent set $V(G) \backslash Q$. As $N^{\prime} \subseteq N$, it follows that $G$ is probe split with respect to the partition $(N, P)$. This settles the first case.

2. $S_{P}(\emptyset) I_{P}$

Let $x \in S_{P}, y \in I_{P}$ with $x y \in E$ such that $\left|N(x) \cap I_{P}\right|+\left|N(y) \cap S_{P}\right|$ is maximum. Partition $S_{P}-x$ and $I_{P}-y$ as:

$$
\begin{aligned}
S_{1} & =\left(S_{P}-x\right) \cap N(y), \\
S_{0} & =\left(S_{P}-x\right) \backslash S_{1}, \\
I_{1} & =\left(I_{P}-y\right) \cap N(x), \\
I_{0} & =\left(I_{P}-y\right) \backslash I_{1} .
\end{aligned}
$$

Then $S_{1}(0) I_{1}$ (otherwise there would be a $\sqrt[\mathfrak{B}_{3}]{\text { }}$ and $S_{0}$ (0) $I_{0}$ (otherwise there would be a $\mathfrak{B}_{1}$. Moreover, $S_{1}$ (0) $I_{0}$ : If $x_{1} \in S_{1}$ is adjacent to $y_{0} \in I_{0}$, then $I_{1}=\emptyset$ (otherwise there would be a $\left[\mathfrak{B}_{1}\right.$, which implies that $\left|N\left(x_{1}\right) \cap I_{P}\right|+\left|N(y) \cap S_{P}\right|>\left|N(x) \cap I_{P}\right|+\left|N(y) \cap S_{P}\right|$, contradicting the choice of $x, y$. Similarly, $I_{1}$ (0) $S_{0}$. Let

$$
\begin{aligned}
& A=\left\{v \in I_{N} \cap N(x) \mid v \text { (1) } C_{P}\right\}, \\
& B=I_{N} \backslash A .
\end{aligned}
$$

Then

$$
A \text { (1) } S_{1} \wedge B(0) S_{0} \wedge S_{N}(0) I_{0}
$$


because if $a \in A$ were adjacent to $s_{1} \in S_{1}$ then $x y a s_{1}$ induce a $\mathfrak{B}_{4}$ if $b \in B$ were adjacent to $s_{0} \in S_{0}$, then by Lem. 30 and definition of $B, b x \notin E$ and thus $x y b s_{0}$ induce a $\mathfrak{B}_{2}$, if $s \in S_{N}$ were adjacent to $i \in I_{0}$ then xysi induce a $\mathfrak{B}_{2}$. Furthermore,

$$
\left|\left(S_{1}+x\right) \cap N(B)\right| \leq 1 \wedge\left|\left(I_{1}+y\right) \cap N\left(S_{N}\right)\right| \leq 1:
$$

If $b \in B$ has two neighbours $s, s^{\prime} \in S_{1}+x$, then $y b s s^{\prime}$ induce a $\mathfrak{B}_{4}$. And if $s, s^{\prime} \in S_{1}+x\left(s \neq s^{\prime}\right)$ have different neighbours $b, b^{\prime} \in B$, respectively, then $b s y s^{\prime} b^{\prime}$ induce a $\mathfrak{B}_{8}$. The proof of the second statement is similar.

We now consider three cases.

(a) $B$ (b) $S_{1}$

By [36, we let $S_{1} \cap N(B)=\left\{s_{B}\right\}$, then $B$ (0) $S_{1}+x-s_{B}$. Let $b \in B$ be adjacent to $s_{B}$. Then

$$
A=\emptyset, \quad I_{1}=\emptyset, \quad y \text { (1) } C,
$$

because otherwise $a x y s_{B} b$, with $a \in A$, would induce a $\mathfrak{B}_{8}, x i_{1} s_{B} b$, with $i_{1} \in I_{1}$, would induce a ${\mathfrak{\mathfrak { B } _ { 2 }}}_{\text {, and }} x y c s_{B}$, with $c \in C$ non-adjacent to $y$, would induce a $\mathfrak{B}_{3}$ or a $\mathfrak{B}_{4}$.

Then $G$ is a split graph with clique $C+s_{B}+y$ and independent set $\left(S-s_{B}\right) \cup(I-y)$, and thus trivially probe split.

(b) $B$ (0) $S_{1} \wedge S_{N}$ (b) $\left(I_{1}+y\right)$

By [36, we let $\left(I_{1}+y\right) \cap N\left(S_{N}\right)=\left\{i_{S}\right\}$, then $S_{N}$ (0) $I_{1}+y-i_{S}$. Then

$$
A(0) S_{0}, \quad i_{S} \text { (1) } C \text {, }
$$

because otherwise $s i_{S} x a s_{0}$, with $a \in A$ adjacent to $s_{0} \in S_{0}$ and $s \in S_{N}$ adjacent to $i_{S}$, would induce a $\mathfrak{B}_{9}$, and $x c s i_{S}$, with $s \in S_{N}$ adjacent to and $c \in C$ non-adjacent to $i_{S}$, would induce a $\mathfrak{B}_{4}$

Then $G$ is a split graph with clique $C+i_{S}+x$ and independent set $(S-x) \cup\left(I-i_{S}\right)$ and thus trivially probe split.

(c) $B$ (0) $S_{1} \wedge S_{N}$ (1) $\left(I_{1}+y\right)$

First assume that $S_{1} \neq \emptyset$. Let $s_{1} \in S_{1}$, then

$$
A \text { (1) } S_{0}, \quad y \text { (1) } C \text {, }
$$

because otherwise $a s_{0} s_{1} y$, with $a \in A$ and $s_{0} \in S_{0}$ neighbours, would induce a $\mathfrak{B}_{2}$, and $x y c s_{1}$, with $c \in C$ non-adjacent to $y$, would induce a $\mathfrak{B}_{3}$ or a $\mathfrak{B}_{4}$

Then $G$ is a split graph with clique $C+x+y$ and independent set $(S-x) \cup(I-y)$ and thus trivially probe split.

Next assume $S_{1}=\emptyset$. If we add all edges between vertices in $A$ then we get a split graph with clique $C+x \cup A$ and independent set $(S-x) \cup(I \backslash A)$. As $A \subseteq N$, if follows that $Q$ is probe split with respect to the partition $(N, P)$.

This settles the second case, and Th. 31 is proved. 
Together, Lem. 30 and Th. 31 prove that partitioned probe split graphs can be recognized in polynomial time:

Theorem 32 Given $G=(N, P, E)$ with $N$ an independent set, it can be decided in $O\left(V^{2}+V E\right)$ time whether $G$ is probe split.

Proof: First check with Alg. 1 that $G$ is probe split and, if so, calculate a valid partition $(C, S, I)$. Next check that Lem. 30 holds and work through Th. 31. This can be done in the desired timebound. 


\section{References}

[1] G. J. Chang, A. J. J. Kloks, J. Liu, and S. L. Peng. The PIGs full monty - A floor show of minimal separators. In Proceedings of 22nd Symposium on Theoretical Aspects of Computer Science (STACS) February 24-26, Stuttgart, Germany. Lecture Notes in Computer Science, volume 3404, pages 521$532,2005$.

[2] G. J. Chang, T. Kloks, and S.-L. Peng. Probe interval bigraphs. In 2nd Brazilian Symposium on Graphs, Algorithms and Combinatorics, 2005.

[3] M.-S. Chang, T. Kloks, D. Kratsch, J. Liu, and S. L. Peng. On the recognition of probe graphs of some self-complementary classes of perfect graphs. In Proceedings of the 11th International Computing and Combinatorics Conference (COCOON) August 16-19 2005, Kunming, Yunnan, China, Lecture Notes in Computer Science, volume 3595, pages 808-817, 2005.

[4] S. Foldes and P. L. Hammer. Split graphs. In Proc. 8th southeast. Conf. on Combinatorics, graph theory, and computing; Baton Rouge, pages 311-315. 1977.

[5] M. C. Golumbic and M. Lipshteyn. Chordal probe graphs. Discrete Appl. Math., 143(1-3):221-237, 2004.

[6] P. L. Hammer and B. Simeone. The splittance of a graph. Combinatorica, 1:275-284, 1981.

[7] A. Hertz. Slim graphs. Graphs Comb., 5(2):149-157, 1989.

[8] C. T. Hoàng and F. Maffray. On slim graphs, even pairs, and star-cutsets. Discrete Math., 105(13):93-102, 1992.

[9] Information System on Graph Classes and their Inclusions (ISGCI).

http://wwwteo.informatik.uni-rostock.de/isgci.

[10] J. L. Johnson and J. P. Spinrad. A polynomial time recognition algorithm for probe interval graphs. In Proceedings of the 12th annual ACM-SIAM symposium on discrete algorithms. Washington, DC, USA, January 7-9, 2001. Philadelphia, PA: Society for Industrial and Applied Mathematics (SIAM), pages 477-486. 2001.

[11] R. M. McConnell and J. P. Spinrad. Construction of probe interval models. In Proceedings of the thirteenth annual ACM-SIAM symposium on discrete algorithms, San Francisco, CA, USA, January 6-8, 2002. Philadelphia, PA: Society for Industrial and Applied Mathematics (SIAM), pages 866875. 2002.

[12] F. R. McMorris, C. Wang, and P. Zhang. On probe interval graphs. Discrete Appl. Math., 88(13):315-324, 1998.

[13] N. Przulj and D. G. Corneil. 2-tree probe interval graphs have a large obstruction set. Discrete Appl. Math., 150(1-3):216-231, 2005.

[14] L. Sheng. Cycle free probe interval graphs. Congr. Numerantium, 140:33-42, 1999.

[15] P. Zhang. Probe interval graphs and its application to physical mapping of DNA. Unpublished, 1994. 